\title{
Composite Material Behavior Using a Homogenization Double Scale Method
}

\author{
S. Oller ${ }^{1}$; J. Miquel Canet ${ }^{2}$; and F. Zalamea ${ }^{3}$
}

\begin{abstract}
In this paper, we present a two-scale numerical method in which structures made up of composite materials are simulated. The method proposed lies within the context of homogenization theory and assumes the periodicity of the internal structure of the material. The problem is divided into two scales of different orders of magnitude: A macroscopic scale in which the body and structure of the composite material is simulated, and a microscopic scale in which an elemental volume called a "cell" simulates the material. In this work, the homogenized strain tensor is related to the transformation of the periodicity vectors. The problem of composite materials is posed as a coupled, two-scale problem, in which the constitutive equation of the composite material becomes the solution of the boundary-value problem in the cell domain. Solving various examples found in the bibliography on this subject demonstrates the validity of the method.
\end{abstract}

DOI: 10.1061/(ASCE)0733-9399(2005)131:1(65)

CE Database subject headings: Composite materials; Constitutive models; Computation; Homogeneity; Composite structures.

\section{Introduction}

Various multiscale methods have been developed to solve the problems presented by the mechanical behavior of composite materials. These methods can be included in the context of homogenization theory, in which the composite material problem is divided into two different scales. Thus, the composite material is assumed to be homogeneous on a macroscopic scale $\left(x_{i}\right)$ and its behavior can be studied by taking a representative unit volume, which is represented on a second scale called the microscopic scale $\left(y_{i}\right)$. When the internal structure of the composite material is periodic, the representative unit volume is called a cell. The use of these two scales is equivalent to determining the properties of a given composite material under a limiting condition, i.e., when the cell's dimensions tend toward zero.

One of the most relevant methods is the homogenization theory proposed and developed by Sanchez-Palencia (1980), Bensoussan et al. (1978), and Duvaut (1976), which is formulated in terms of the asymptotic expansion theory. Due to their work, the

\footnotetext{
${ }^{1}$ Departamento de Resistencia de Materiales y Estructuras en la Ingeniería, Univ. Politécnica de Cataluña. Jordi Girona 1-3, Módulo C1, Campus Norte UPC, 08034 Barcelona, Spain (corresponding author). E-mail: sergio.oller@upc.es

${ }^{2}$ Departamento de Resistencia de Materiales y Estructuras en la Ingeniería, Univ. Politécnica de Cataluña. Jordi Girona 1-3, Módulo C1, Campus Norte UPC, 08034 Barcelona, Spain. E-mail: canet@cimne.upc.es

${ }^{3}$ Departamento de Resistencia de Materiales y Estructuras en la Ingeniería, Univ. Politécnica de Cataluña. Jordi Girona 1-3, Módulo C1, Campus Norte UPC, 08034 Barcelona, Spain. E-mail: zalamea@cimne.upc.es

Note. Associate Editor: Henry K. Stolarski. Discussion open until June 1, 2005. Separate discussions must be submitted for individual papers. To extend the closing date by one month, a written request must be filed with the ASCE Managing Editor. The manuscript for this paper was submitted for review and possible publication on October 29, 2002; approved on July 20, 2004. This paper is part of the Journal of Engineering Mechanics, Vol. 131, No. 1, January 1, 2005. @ASCE, ISSN 0733 9399/2005/1-65-79/\$25.00.
}

division of the problem into two scales rests on a rigorous theoretical base. Suquet $(1982,1987)$, on the other hand, uses the method of averages and finds that upon extending it to the nonlinear case, the macroscopic variables become coupled to the respective microscopic ones. This fact presents a significant difficulty in considering the two-scale coupling, because it implies that the composite's constitutive equation depends on an "infinite number of internal variables." For the purposes of obtaining a simplified constitutive law for the composite, certain simplifications have been proposed, although these are only applicable to simpler structural problems. In recent years, various methods have been proposed as a solution to nonlinear two-scale problems. Ghosh (1996) proposes a nonconventional method in which he represents the composite's microstructure using finite Voronoi elements, and he uses the asymptotic expansion theory as a bridge between the two scales. Fish et al. (1997) use the field transformation theory (Dvorak et al. 1994), as well as asymptotic expansion theory. In all cases, the use of these methods seeks to reduce computational effort, although it may also result in a loss of precision.

In this work, we propose a double-scale method using standard continuum mechanics, in which we hold that the values of the macroscopic variables derive from the hypothesis that the composite's microstructure is periodic. Thus, the auxiliary tensors, known as concentration or localization tensors, are not used. The solution is arrived at by means of the numerical coupling of the two scales using the finite element method.

\section{Periodic Structure Division}

The periodic distribution of the constituent materials creates certain symmetries, which allow us to divide the composite material into structural units called cells. This virtual division of the composite is achieved by means of material surfaces, which we shall call sides. Thus, a composite material represented as a twodimensional space can be divided into cells of four sides (two pairs of periodic sides) called quadrilateral cells, or into cells of six sides (three pairs of periodic sides) called hexagonal cells. The 


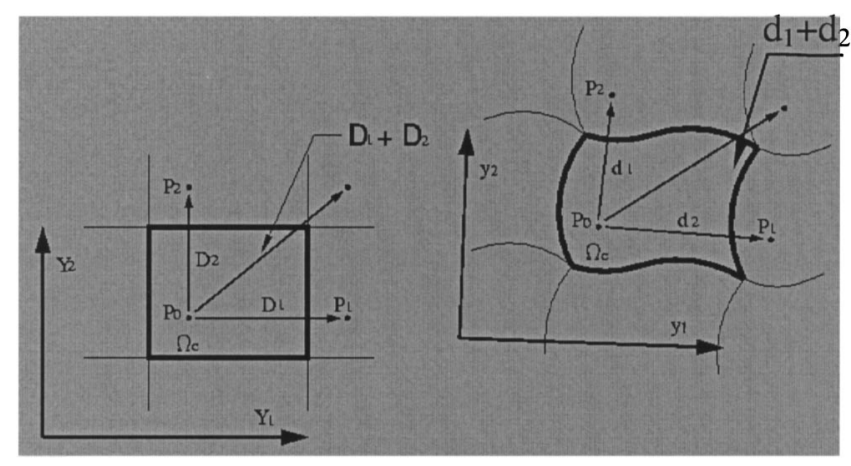

Fig. 1. Periodicity vectors in the $\mathbf{D}$ configuration and the new periodicity vectors $\mathbf{d}$ on the updated configuration of cell domain $\Omega_{c}$

points at which the material coordinates $X_{i}$ have the same relative position in the neighboring cells are called periodic points. For example, in Fig. 1, any point in the cell domain is indicated by $P$; in the neighboring cells, these same points are indicated, and these are called periodic points. The relative position between said points determines the base of the vectors $\mathbf{D}$ that will henceforth be referred to as periodicity vectors (also known as the cell's base vectors); they possess specific dimensions and directions related to the cell domain.

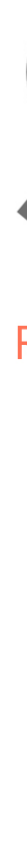

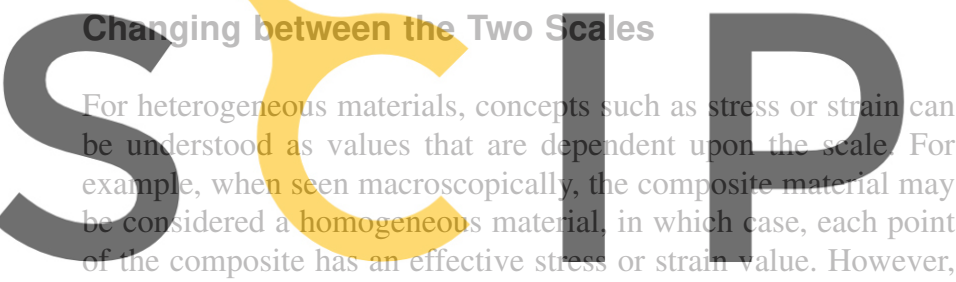
at the microscopic level, there may be considerable fluctuations

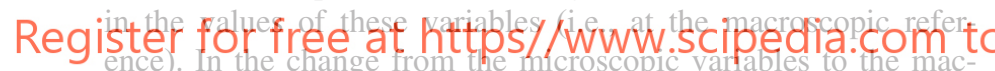
roscopic ones, the hypothesis of local periodicity is used (Sanchez-Palencia 1987). This hypothesis is a consequence of the minimization of energy, as a result of which the microscopic variables within the cell are equal to the respective variables of neighboring cells but may be very different from those of more distant cells.

\section{Homogenized Strain Tensor}

Consider that $\Omega_{c}$ represents a cell domain of a composite material (represented in material space as $Y_{i}$; see Fig. 1) and that this cell domain is characterized by the periodicity vector $\mathbf{D}=\mathbf{Y}_{p}-\mathbf{Y}_{p 0}$. If this domain then undergoes displacement, the local periodicity hypothesis ensures that the composite material, despite its consequent deformation, retains its periodicity relationship with its neighboring cells. In other words, the deformed cells, together with their neighboring cells, all undergo the same transformation. Consequently, the new periodicity vectors $\mathbf{d}$ may be written as

$$
\mathbf{d}=\mathbf{y}_{p}-\mathbf{y}_{p 0}=\mathbf{D}+\left(\mathbf{u}_{P}-\mathbf{u}_{P 0}\right)
$$

where $\mathbf{u}_{P}-\mathbf{u}_{P 0}=$ difference in displacement between the periodic points. The transformation of the space of the cells is associated with the change of periodicity vectors. The partial derivative of these vectors is

$$
\frac{\partial \mathbf{d}}{\partial \mathbf{D}}=\frac{\partial\left(\mathbf{y}_{p}-\mathbf{y}_{p 0}\right)}{\partial\left(\mathbf{Y}_{p}-\mathbf{Y}_{p 0}\right)}
$$

Now, on the macroscopic level, the periodicity vectors are infinitesimally small $(|\mathbf{D}| \rightarrow 0)$. Consequently, on a macroscopic scale, this value tends toward a limit

$$
\lim _{D \rightarrow 0}\left[\frac{\partial \mathbf{d}}{\partial \mathbf{D}}\right]=\lim _{D \rightarrow 0}\left[\frac{\partial\left(\mathbf{y}_{p}-\mathbf{y}_{p 0}\right)}{\partial\left(\mathbf{Y}_{p}-\mathbf{Y}_{p 0}\right)}\right]=\frac{\partial \mathbf{x}}{\partial \mathbf{X}}=\mathbf{F}
$$

Therefore,

$$
\mathbf{d}=\mathbf{F D}
$$

where $\mathbf{F}=$ homogenized deformation gradient tensor. This simple change of scale allows us to obtain the macroscopic strain tensor using classical continuum mechanics. The square of the length of the new periodicity vectors is

$$
|\mathbf{d}|^{2}=\mathbf{D}^{\mathbf{T}} \mathbf{F}^{\mathbf{T}} \mathbf{F D}
$$

and the difference between the square of the length of the new periodicity vectors and the periodicity vectors in the reference configuration is

$$
\begin{gathered}
|\mathbf{d}|^{2}-|\mathbf{D}|^{2}=\left[\mathbf{D}^{\mathrm{T}} \mathbf{F}^{\mathrm{T}}\right][\mathbf{F} \mathbf{D}] \mathbf{D}^{\mathrm{T}} \mathbf{D} \\
|\mathbf{d}|^{2}-|\mathbf{D}|^{2}=2 \mathbf{D}^{\mathrm{T}} \widetilde{\mathbf{E}} \mathbf{D}
\end{gathered}
$$

Thus, the Green Lagrange tensor is obtained as a measure of

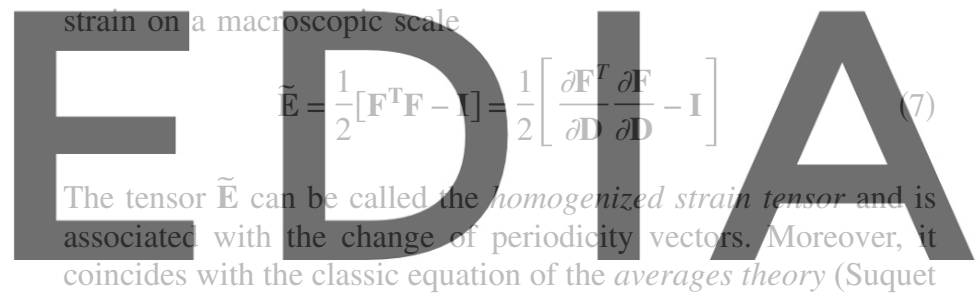
1982):

\section{download the version without the watermark

$$
\widetilde{\mathbb{E}}=\langle\mathbb{E}(y)\rangle_{\Omega_{c}}=\frac{1}{V_{c}} \int_{V_{c}} \mathbb{E}(y) d V_{c}
$$

where $\langle\mathbf{E}(y)\rangle_{\Omega_{c}}=$ microscopic strain field; $\Omega_{c}=$ domain of the cell; and $V_{c}=$ volume contained in $\Omega_{c}$.

\section{Homogenized Stress Tensor and Equilibrium Equation}

The Cauchy equilibrium equation on the microscopic scale, at cell level, may be written as

$$
\int_{S_{c}} \sigma_{i j} n_{j} d S=\int_{V_{c}} \rho a_{i} d V-\int_{V_{c}} \rho b_{i} d V
$$

The domain of the cell is very small from the macroscopic point of view $\left(V_{c} \rightarrow 0\right)$. Consequently, the value of the forces of volume and inertia are also small and tend to zero. This equation ensures equilibrium in the domain of the cell:

$$
\lim _{V_{c} \rightarrow 0}\left(\int_{S_{c}} \sigma_{i j} n_{j} d S\right)=\lim _{V_{c} \rightarrow 0}\left(\int_{V_{c}}\left(\rho a_{i}-\rho b_{i}\right) d V\right)=0
$$

where $S_{c}=$ boundary surface of the cell; $\sigma_{i j}=$ microscopic stress field; and $n_{j}=$ unitary normal vector of the surface element $d S$. Observe that the orientation of the two surface elements located at 


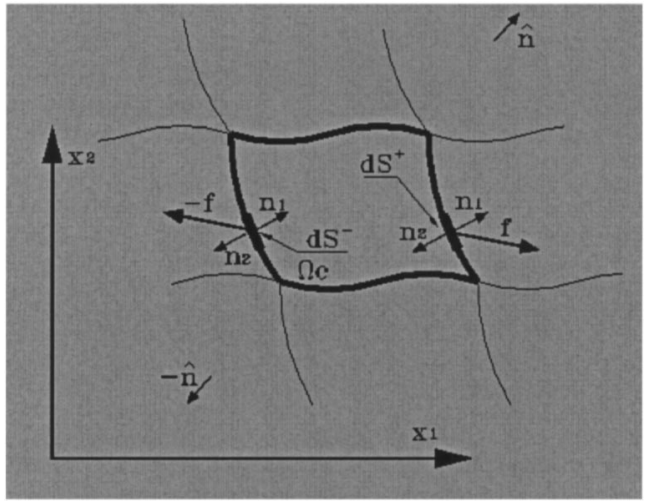

Fig. 2. Forces acting on the cell boundaries are equal but in opposite directions
periodic points (see Fig. 2) has unitary normal vectors $\left(\mathbf{n}_{1}\right.$ and $\mathbf{n}_{2}$ ) in opposite directions. The principle of action and reaction en- sures that the surface forces $\mathbf{f}=\mathbf{t}(\mathbf{n}) d S_{c}$ in the two surface ele- ments are equal but in opposite directions. This is known in the literature on homogenization as an antiperiodic force field on the cell's sides (Lene 1986).
A second-order tensor $\widetilde{\sigma}_{j}$ is defined as the average of the forces acting on the cell's sides:
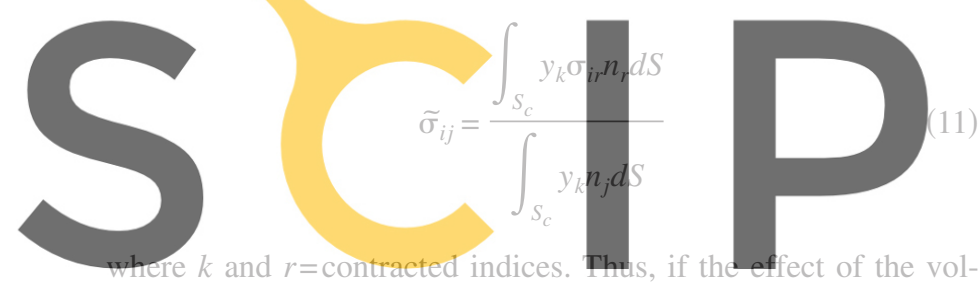

ume forces is disregarded $\left(\sigma_{i j, j}=0\right)$, using the divergence theorem

\section{Register for free at hiteps/\%ww.scipedia.com to}

$$
\widetilde{\sigma}_{i j}=\frac{\int_{V_{c}} y_{k, i} \sigma_{i r} d V}{\int_{V_{c}} y_{k, j} d V}=\frac{\int_{V_{c}} \delta_{k, i} \sigma_{i r} d V}{\int_{V_{c}} y_{k, j} d V}=\frac{1}{V_{c}} \int_{V_{c}} \sigma_{i j} d V
$$

The overall surface force $\widetilde{\mathbf{t}}(\hat{\mathbf{n}})$ is defined as the average of the forces on the cell sides $S_{c}$, that is determined by the direction of a (macroscopic) unitary vector $\hat{\mathbf{n}}$,

$$
\tilde{t}(\hat{\mathbf{n}})=\left[\frac{\int_{S_{c}} y_{k} \sigma_{r} n_{r} d S}{\int_{S_{c}} y_{k} n d S}\right] \hat{n}_{k}=\left[\frac{1}{V_{c}} \int_{V_{c}} \sigma d V\right] \hat{n}_{k}
$$

Because the value of the right-hand side of the equation is a linear function that depends on the direction of vector $\hat{\mathbf{n}}$ (i.e., $\widetilde{t}_{i}(\hat{\mathbf{n}})$ $=\widetilde{\sigma}_{i k} \hat{\mathbf{n}}_{k}$ ), and since the tensor $\widetilde{\sigma}$ fulfills, on the macroscopic level, the same requirements as the stress tensor $\boldsymbol{\sigma}$ (in homogeneous materials), tensor $\tilde{\boldsymbol{\sigma}}$ is henceforth called the homogenized stress tensor.

Furthermore, if the entire composite material's domain $\Omega$ (which is formed by an infinite number of cells) is considered, the static equilibrium equation may be written as follows:

$$
\int_{S}\left[\frac{1}{V_{c}} \int_{V_{c}} \sigma_{i j} d V\right] \hat{n}_{j} d S+\int_{V}\left[\frac{1}{V_{c}} \int_{V_{c}} \rho b_{i} d V\right] d V=0
$$

Obviously, the body forces per unit volume have been considered (since on a macroscopic scale their magnitudes can be significant). This effect may be assumed as the average of the volume forces $(\tilde{\mathbf{b}})$ inside the cell, or

$$
\tilde{b}_{i}=\frac{1}{V_{c}} \int_{V_{c}} \rho b_{i} d V=\text { constant }
$$

Substituting Eqs. (11) and (14) in Eq. (13), and transforming the surface integral by using the divergence theorem, results in

$$
\int_{V_{c}} \tilde{\sigma}_{i j, j} d V+\int_{V_{c}} \tilde{b}_{i} d V=0
$$

Eq. (16) is valid for any arbitrary volume $V$; therefore, it is also valid when choosing very small domains [being the limit the cell domain $\left.\left(\Omega \rightarrow \Omega_{c} ; \Omega_{c} \rightarrow 0\right)\right]$. Then,

$$
\widetilde{\sigma}_{i j, j}+\tilde{b}_{i}=0
$$

and thus we obtain the homogenized local equation of static equilibrium.

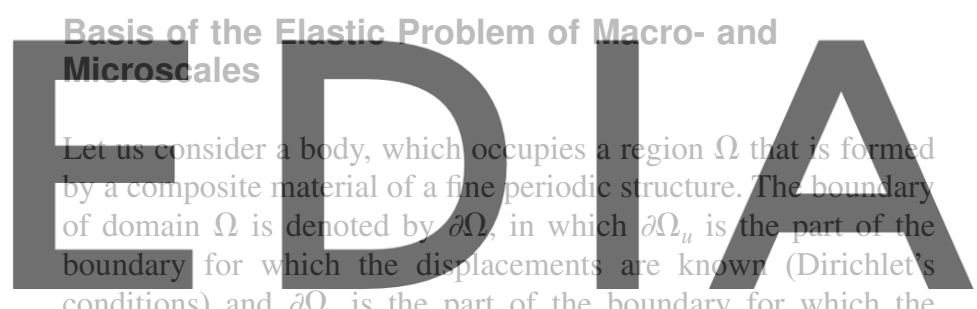
conditions) and $\partial \Omega_{t}$ is the part of the boundary for which the surface forces are known (Newman's condition). The structure of dow mkoad athellversio pe without the wotetermalk units (cells). This domain is denoted by space $\Omega_{c}$, such that, by ordered repetition, the body of the composite material $\Omega$ can be restored. Míoreover, two scales of differing orders of magnitude are assumed, in such a way that the particles of domain $\Omega$ can be labeled in accordance with their position within a space $x_{i}$ (at a macroscopic level), while the position of the cell's particles can be labeled within a local space $y_{i}$ (at a microscopic level). Therefore, at the macroscopic level, the problem of composite materials becomes a boundary-value problem of homogeneous materials, in which we look for displacement and stress fields $[\mathbf{u}(x), \widetilde{\boldsymbol{\sigma}}(x)]$ that satisfy the following equations:

$$
\begin{aligned}
& \text { Macroscopic scale: } \\
& \begin{cases}\frac{\partial \widetilde{\boldsymbol{\sigma}}(x)}{\partial \mathbf{x}}+\widetilde{\mathbf{b}}=0 & \text { equilibrium equation in } \Omega \\
\widetilde{\boldsymbol{\sigma}}(x)=\frac{1}{V_{c}} \int_{V_{c}} \boldsymbol{\sigma}(x, y) d V_{c} & \text { constitutive equation in } \Omega \\
\mathbf{u}(x)=\overline{\mathbf{u}}(x) & \text { displacements on } \partial \Omega_{u} \\
\widetilde{\boldsymbol{\sigma}}(x): \mathbf{n}=\overline{\mathbf{t}}(x) & \text { forces on } \partial \Omega_{t}\end{cases}
\end{aligned}
$$

On the other hand, at the microscopic level, the cell boundary conditions must reproduce the material microstructure conditions locally. The periodicity vectors obtained under microscopic strain 
are then formulated in agreement with the local periodicity hypothesis

$$
\mathbf{d}-\mathbf{D}=\mathbf{u}_{p}-\mathbf{u}_{p 0}
$$

where $\mathbf{u}_{p}$ and $\mathbf{u}_{p 0}$ represent the displacement of two periodic points; $\mathbf{D}=$ periodicity vector defined between these points in the microscopic reference configuration $X_{i}$; and $\mathbf{d}=$ new periodicity vector resulting from the strain field (see Fig. 1). In the case of small strains, if only the symmetric part of the velocity gradient tensor, called the deformation rate tensor (or stretching tensor), is considered and the influence of the antisymmetric part of the velocity gradient tensor, called the spin tensor (or vorticity tensor), can be rejected, the relative displacement between the cell boundary's periodic points can be approximated by (Zalamea et al. 1999a)

$$
\mathbf{u}_{p}-\mathbf{u}_{p 0} \cong \mathbf{E} \cdot \mathbf{D}
$$

This relative displacement condition between boundary points represents a field of periodic displacements. Additionally, the forces that develop on the cell boundary have the same modulus but in opposite directions. Then, the problem on a microscopic scale, $y_{i}$, is reduced to solving the following boundary-value problem in the cell domain $\Omega_{c}$ :
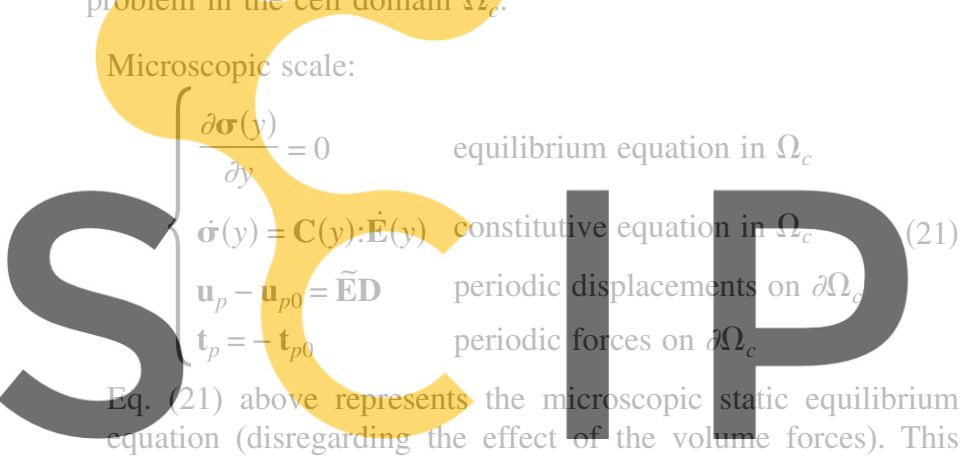
equation must hold for each and every point of the cell domain

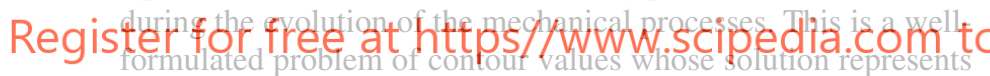
the equilibrium of forces at the microscopic level. This equilibrium represents the balance of forces inside the domain with regard to the boundary cell forces.

The behavior of the component materials is represented by the expression in the second equation in Eq. (21), in which $\mathbf{C}$ is the local constitutive tensor. In this case, the constitutive equation of component materials may represent any kind of mechanical behavior (elastic, plastic, viscous, etc.). In Eq. (21), we can see the displacement and periodic force conditions in the boundary of the cell $\partial \Omega_{c}$, which are at the same time related to the strain tensor $\widetilde{\mathbf{E}}$ at the macroscopic level. Consequently, the two problems are coupled, and therefore, the solution of the problem on the macroscale is obtained following a classic procedure, that is, using the equilibrium equation in a discrete solid (see Zienkiewicz and Taylor 1991), which requires that the microscopic boundary-value problem conditions be satisfied in each point of the macro domain $\Omega$. This task involves an infinite number of problems at the microscopic level. However, despite the difficulties involved, the microscopic problem can be solved in a discrete way, by using the finite element method to write the elastic microscopic equilibrium equation (Zienkiewicz and Taylor 1991):

$$
\mathbf{f}^{\text {int }}(\boldsymbol{\sigma})=\mathbf{f}^{\text {ext }}=\mathbf{f} \Rightarrow \mathbf{K} \cdot \mathbf{U}=\mathbf{f}
$$

where $\mathbf{K}=$ assembled stiffness matrix at cell level; $\mathbf{U}=$ nodal displacement vector; and $\mathbf{f}=$ force vector in the boundary of the cell.
There are several ways of solving this equilibrium equation under periodic boundary conditions, although, in this work, the Lagrange Multiplier Method is used $(\boldsymbol{\lambda})$. However, the latter has several disadvantages, such as an increase in the number of equations and bandwidth of the stiffness matrix, as well as the null terms that are produced in the diagonal of the stiffness matrix, which can create computation difficulties. The latter can be solved by implementing the contour conditions imposed by Anthoine (1995), who divided the Lagrange multipliers into two groups, $\boldsymbol{\lambda}_{1}$ and $\boldsymbol{\lambda}_{2}$, which represent the forces in the respective periodic nodes of the boundary of a cell, and whose magnitude depends on the relative displacement of the boundary nodes of this cell. The stationary solution of the augmented functional with Lagrange multipliers is as follows:

$$
\begin{aligned}
\Pi= & \frac{1}{2} \mathbf{U}^{T} \cdot \mathbf{K} \cdot \mathbf{U}-\mathbf{U}^{T} \cdot \mathbf{f}+\lambda_{1}^{T} \cdot\left(\mathbf{k}_{p} \cdot \mathbf{U}-\mathbf{U}_{\mathbf{r}}\right)+\lambda_{2}^{T} \cdot\left(\mathbf{k}_{p} \cdot \mathbf{U}-\mathbf{U}_{\mathbf{r}}\right) \\
& +\frac{1}{2}\left(\lambda_{1}-\lambda_{2}\right)^{T} \cdot\left(\lambda_{1}-\lambda_{2}\right)
\end{aligned}
$$

This leads to the following well-conditioned linear equation system:
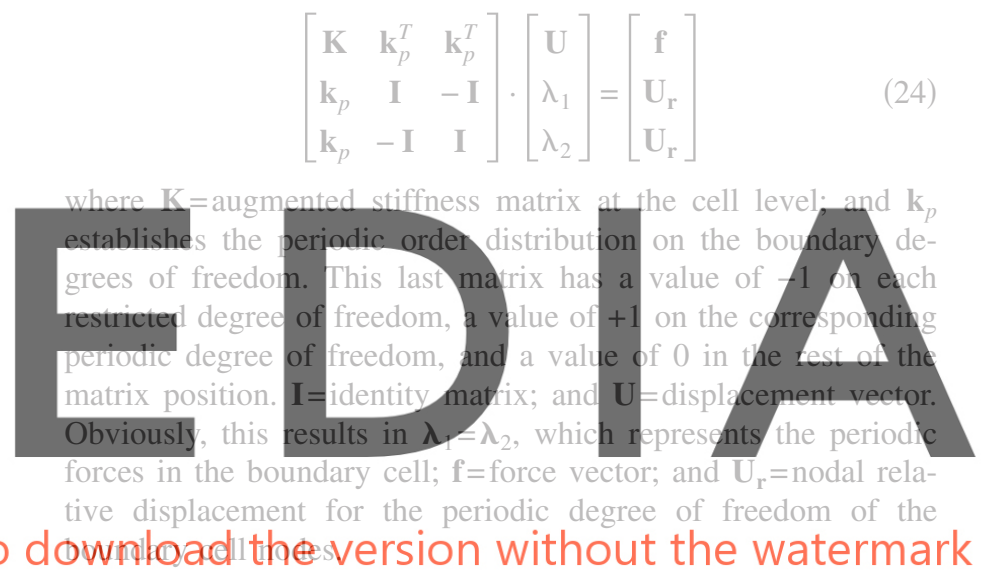

\section{Basis of the Inelastic Problem of Macro- and Microscales}

A linear and nonlinear range characterizes the behavior of composite materials. Consequently, in a loaded composite solid, it is possible that one or more compounding materials may reach the elastic threshold, developing plasticity, degradation, fractures, etc., at the microstructural level. In each of these cases, the whole composite material behaves in a nonlinear manner. Nonlinear behavior in homogenization theory was introduced by Suquet (1982, 1987). The writer concludes that "the behavior of the composite depends on an infinite number of internal variables," which entails great difficulties due to the enormous computational effort involved. In later works, the writer makes certain peculiar simplifications taking into account the nature of each component material (see, for example, Lene 1986; Suquet 1987; Devries et al. 1989). The aforementioned writers also devise certain constitutive equations for certain compound materials (Aravas et al. 1995). In all of these cases, the proposals are approximate and only applicable to particular composites, and they cannot be generalized.

It was Fish who proposed the first solution (Fish et al. 1997; Fish and Shek 1999), using the theory of asymptotic expansion to obtain the governing equations in each of the two scales. In this case, the behavior of the composite results of solving a cell for 
each numerical integration point. However, to reduce the computational cost, not all the cells are solved in a nonlinear manner; using an algorithm, the nonlinear behavior points of the macroscale are identified depending on their stress/strain levels, and only the cells associated with these points are solved. Also, the averages of the internal variables are only stored for each of the cells displaying nonlinear behavior. This brings a certain loss of precision in the nonlinear resolution of problems.

A second proposal based on the theory of asymptotic development was presented by Ghosh (Ghosh et al. 1996; Lee et al. 1999), in which the behavior of the composite is obtained using Voronoi's finite elements, which allow an entire cell to be represented by just one of these finite elements. The finite elements provide correct results at the macro- and microscale level; nevertheless, in their interior, the microstructure's details are not described.

The present work shows an alternative to the two formulations mentioned above. The conceptual bases have been partially presented in various publications (Zalamea et al. 1998, 1999a,b,

2000, 2002; Zalamea 2001). The theory is based on the direct formulation of governing equations in each scale (see the section entitled, "Basis of the Elastic Problem of Macro- and Microscales" for the elastic formulation), which renders the use of the theory of asymptotic expansion unnecessary. A further characteristic is that the analysis of each scale is carried out by the finite element method and by imposing the boundary conditions in the microseale by means of the Lagrange multipliers [Eqs. (23) and (24)]. Also, during the linearization of nonlinear problems, the equilibrium equation in the macroscales is solved using one cell for each integration point, although tused to prevent high computationa linearization of the nonlinear problen each cell is solved plus an entire co internal variables

The nonlinear solution that uses the linearization procedure

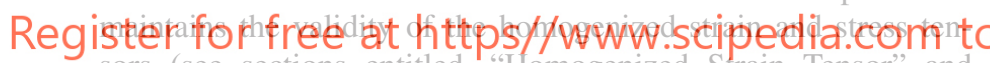
sors (see sections entitled, "Homogenized Strain Tensor" and "Homogenized Stress Tensor and Equilibrium Equation") and the governing equations formulated in the macro- and microscales (see section entitled, "Basis of the Elastic Problem of Macro- and Microscales"). However, the microscale definition of the constitutive equation should be changed [Eq. (21)], to take into account the nonlinear behavior of the material (e.g., damage, plasticity, viscoelasticity, viscoplasticity, fractures, etc.). A generic nonlinear constitutive equation at the microscale level is summarized in the following expression:

Free energy for the single compounding material,

$$
\Phi=\Phi(\mathbf{E}, \alpha)
$$

Free variable: Strain tensor,

$$
\mathbf{E}=\nabla^{S} \mathbf{u}
$$

Internal variables,

$$
\alpha=\left\{\alpha_{k}\right\}, \quad \text { con } k=1, \ldots, n
$$

Dependent variable: Stress tensor,

$$
\dot{\boldsymbol{\sigma}}=\dot{\boldsymbol{\sigma}}(\Phi, \dot{\mathbf{E}}, \alpha)
$$

The constitutive equation for the composite at the current time $t$ $+\Delta t$ is expressed in the following incremental expression that coincides strictly with Eq. (12):

$$
\begin{aligned}
\widetilde{\boldsymbol{\sigma}}^{t+\Delta t} & =\frac{1}{V_{c}} \int_{V_{c}} \boldsymbol{\sigma}^{t+\Delta t} d V=\frac{1}{V_{c}} \int_{V_{c}}\left(\boldsymbol{\sigma}^{t}+\dot{\boldsymbol{\sigma}}^{t+\Delta t} \Delta t\right) d V \\
& =\widetilde{\boldsymbol{\sigma}}^{t}+\left\{\widetilde{\mathbf{C}}^{\mathbf{T}}\right\}^{t+\Delta t}: \dot{\tilde{\mathbf{E}}}^{t+\Delta t} \Delta t
\end{aligned}
$$

where $\left\{\widetilde{\mathbf{C}}^{\mathbf{T}}\right\}^{t+\Delta t}$ represents the homogenized tangent constitutive tensor at the time $t+\Delta t\left(\dot{\tilde{\boldsymbol{\sigma}}}^{t+\Delta t}=\left\{\widetilde{\mathbf{C}}^{\mathbf{T}}\right\}^{t+\Delta t}: \dot{\widetilde{\mathbf{E}}}^{t+\Delta t}\right)$, during the linearization step of the equilibrium equation. Once the homogenized stress tensor is obtained in each point $\widetilde{\boldsymbol{\sigma}}^{t+\Delta t}$, the nonlinear process for the solution of the equilibrium equation is followed in the traditional way.

The nonlinear equilibrium equation of the solid using the finite element procedure can be written as unbalanced forces between the internal $f_{k}^{\text {int }}$ and external $f_{k}^{\text {ext }}$ forces:

$$
0=\underset{\Omega^{e}}{\mathbf{A}}\left\lfloor f_{k}^{\mathrm{int}}-f_{k}^{\mathrm{ext}}\right\rfloor_{\Omega^{e}}=\left.\Delta f_{k}\right|_{\Omega}
$$

In this case, $\mathbf{A}_{\Omega}$ e represents the assembling operator from the elementary level forces to the whole structure (Zienkiewicz and Taylor 1991). These unbalanced forces can be eliminated during the linearization stage $(i+1)$ at time $t+\Delta t$, in the neighborhood of the current equilibrium force state. For this purpose, it is necessary to enforce the equilibrium balance in this current state ( $i$ +1 ) and express this condition by means of a Taylor series expansion truncated in its first variation, which leads to the following:

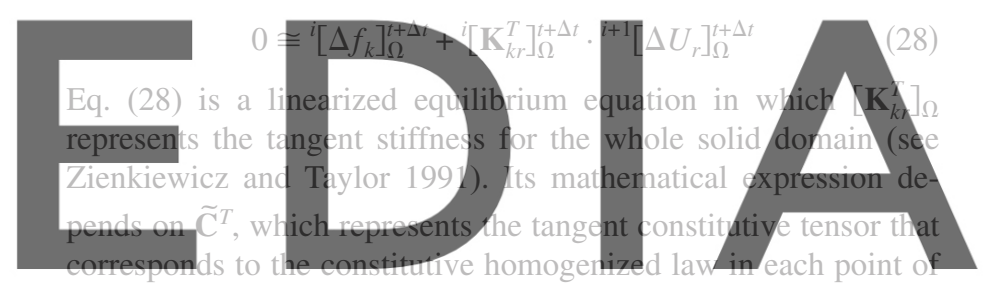

the composite solid. The unbalanced or residual force in the solid

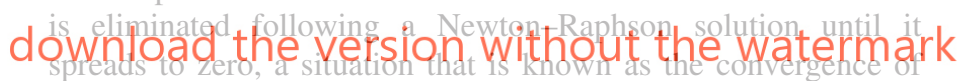

the nonlinear process toward the real solution.

Thus, in the present work, we approached the solution using the numerical finite element method. The result is a general method in which the macroscopic constitutive equation depends exclusively on the microscopic fields.

\section{Determination of the Elastic Constitutive Tensor for Composite Materials}

The global elastic constitutive law for composites, in which there are no slips between the components, can be written as

$$
\widetilde{\boldsymbol{\sigma}}(x)=\widetilde{\mathbf{C}}(x): \widetilde{\mathbf{E}}(x)
$$

Starting from a strain $\widetilde{\mathbf{E}}$, the elastic behavior of the material is obtained through the elastic homogenized constitutive tensor $\widetilde{\mathbf{C}}(x)$. This is a fourth-order tensor and has 81 components. Suquet assumes that the elastic homogenized constitutive tensor shows the classic symmetries if the composite has the periodic distribution of its component:

$$
\widetilde{C}_{i j k l}=\widetilde{C}_{j i l k}=\widetilde{C}_{k l i j}
$$

The property of this material makes the problem easier to solve. In fact, for the orthotropic elastic material, one must only obtain 
nine independent elements of the elastic constitutive tensor. The macroscopic stress tensor $\tilde{\boldsymbol{\sigma}}$ can be obtained directly from the global strain $\widetilde{\mathbf{E}}$, using the elastic homogenized constitutive tensor $\widetilde{\mathbf{C}}(x)$, which is defined by means of the cell information of the composite material.

The method designed to obtain these elastic constants is related to the procedure followed, both for the theory of averages and in asymptotic expansion theory. It allows different perturbations (small displacements) to be applied to the cell in order to activate the different elastic constants of the composite. Since it is not possible to solve the expression $\widetilde{\mathbf{C}}(x)=\widetilde{\boldsymbol{\sigma}}(x) \otimes[\widetilde{\mathbf{E}}(x)]^{-1}[$ because $\widetilde{\mathbf{C}}(x)$ is a fourth-order tensor, while $\widetilde{\boldsymbol{\sigma}}(x)$ and $\widetilde{\mathbf{E}}(x)$ are two second-order tensors, and it therefore has an infinite number of solutions], the homogenized constitutive tensor for the composite material in any cell is obtained by the perturbation procedure. The problem is considered as a system of equations on principal stress directions: A different displacement perturbation is applied in each of the directions and in this case, a unique solution for the composite constitutive tensor is obtained.

For instance, the elastic constitutive tensor can be written, in two-dimensional problems, in plane stress or plane strain as
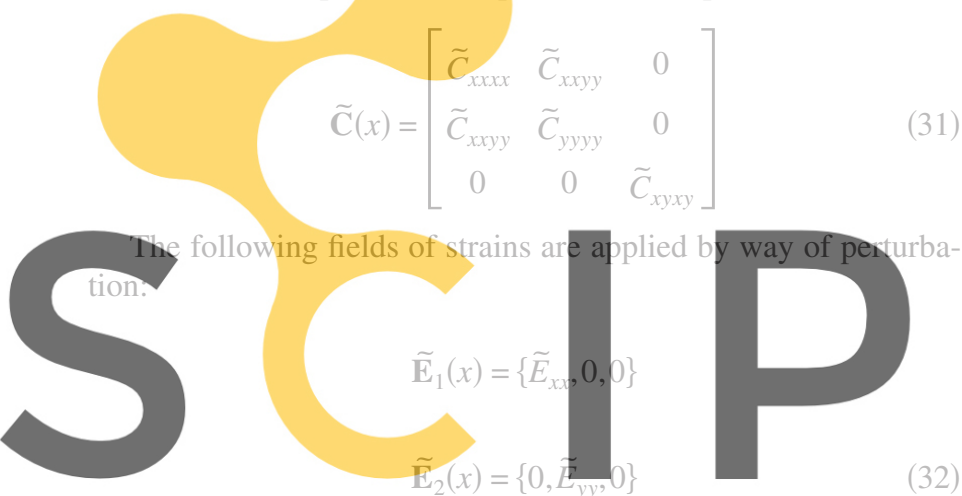

Consequently, if we apply other strain fields to the cell inside the elastic range and the homogenized stress tensor is obtained, this tensor coincides with the homogenized stress preceded by the constitutive homogenized elastic tensor.

\section{Analytical Determination of the Quasi-Tangential Inelastic Constitutive Tensor for Composite Materials}

In nonlinear problems, a tangential or algorithmic homogenized constitutive tensor is required to allow convergence with the smallest possible number of iterations. However, the algorithmic constitutive tensor is quite difficult to obtain for each kind of composite material cell. An incremental iterative method for solving the nonlinear system of the equilibrium equation, such as the modified Newton-Raphson method, can be applied using the elastic constitutive tensor $\widetilde{\mathbf{C}}(x)$ [Eq. (31)], although the computational effort is enormous. Nevertheless, an alternative way can be found which produces quite satisfactory results. In this paper, a method based on the "quasi-tangential" homogenized constitutive tensor is used to obtain the stiffness, a quasi-tangential $\mathbf{K}^{T}$ for the macrostructure, from the values obtained from the previous iteration. This operation may be carried out in various ways (Dennis and More 1977; Crisfield 1980). In this paper, we present an approximate method to obtain the constitutive tensor of the tangential composite material at each point of the solid. We suppose that the

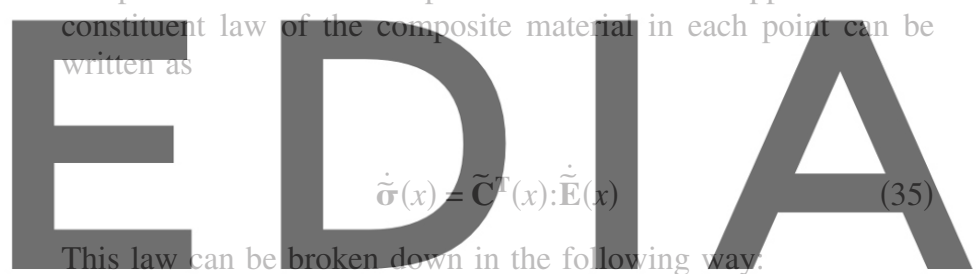

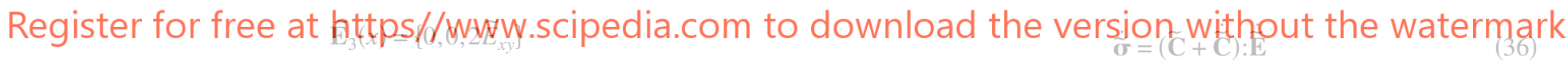

For each of these strain fields, the homogenized stress tensor is obtained: $\widetilde{\boldsymbol{\sigma}}\left(\widetilde{\mathbf{E}}_{1}(x)\right), \widetilde{\boldsymbol{\sigma}}\left(\widetilde{\mathbf{E}}_{2}(x)\right), \widetilde{\boldsymbol{\sigma}}\left(\widetilde{\mathbf{E}}_{3}(x)\right)$. Next, the coefficients of the constitutive tensor are obtained, which are, in this case, the following:

$$
\begin{aligned}
& \widetilde{C}_{x x x x}=\tilde{\boldsymbol{\sigma}}_{x x}\left(\widetilde{\mathbf{E}}_{1}(x)\right) / \widetilde{E}_{x x} \\
& \tilde{C}_{x x y y}=\widetilde{\boldsymbol{\sigma}}_{x x}\left(\widetilde{\mathbf{E}}_{2}(x)\right) / \widetilde{E}_{y y} \\
& \tilde{C}_{y y y y}=\widetilde{\boldsymbol{\sigma}}_{y y}\left(\tilde{\mathbf{E}}_{2}(x)\right) / \widetilde{E}_{y y} \\
& \tilde{C}_{y y x x}=\tilde{\boldsymbol{\sigma}}_{y y}\left(\tilde{\mathbf{E}}_{1}(x)\right) / \widetilde{E}_{x x} \\
& \widetilde{C}_{x y x y}=\widetilde{\boldsymbol{\sigma}}_{x y}\left(\widetilde{E}_{3}(x)\right) / 2 \widetilde{E}_{x y}
\end{aligned}
$$

The analytical demonstration of this constitutive tensor symmetry hypothesis is not trivial. The various writers who have explained the asymptotic expansion theory have presented this demonstration. Using this numerical implementation, or periodicity function, the following symmetry hypothesis is satisfied:

$$
\widetilde{C}_{y y x x}=\widetilde{C}_{x x y y}
$$

where $\widetilde{\mathrm{C}}=$ elastic composite material's constitutive tensor; and $\dot{\widetilde{\mathbf{C}}}=$ rate of this elastic tensor that is to be fulfilled by the tangential condition of the constitutive tensor $\widetilde{\mathbf{C}}^{\mathbf{T}}$. Therefore, the following expression of $\dot{\widetilde{\mathbf{C}}}$ can be obtained from Eq. (36):

$$
\dot{\widetilde{\boldsymbol{\sigma}}}-\widetilde{\mathbf{C}}: \dot{\widetilde{\mathbf{E}}}=\dot{\widetilde{\mathbf{C}}}: \dot{\widetilde{\mathbf{E}}} \Rightarrow \dot{\widetilde{\mathbf{C}}}=(\dot{\widetilde{\boldsymbol{\sigma}}}-\widetilde{\mathbf{C}}: \dot{\widetilde{\mathbf{E}}}) \otimes \dot{\widetilde{\mathbf{E}}}^{-1}
$$

For the reason stated in the previous section, Eq. (37) above has infinite solutions. Nevertheless, because all that we want to obtain is an approach to the tangential constitutive tensor $\widetilde{\mathbf{C}}^{\mathbf{T}}$, several additional restrictions to the rate constitutive tensor $\dot{\widetilde{\mathbf{C}}}$ can be imposed to diminish the indetermination degree and obtain a unique solution. To this end, we suppose that the rate tensor $\dot{\widetilde{\mathbf{C}}}$ maintains the same symmetries as the elastic tensor $\widetilde{\mathbf{C}}$ [see Eq. (30)]; we also suppose that many of the terms are null [see the equivalence with Eq. (31)]. In this case, the solution can easily be obtained. In this work, $\dot{\tilde{\mathbf{C}}}$ is obtained by the procedure described for the determination of the elastic constitutive tensor of the composite material $\widetilde{\mathbf{C}}$ (see section entitled, "Determination of the Elastic Constitutive Tensor for Composite Materials"). 


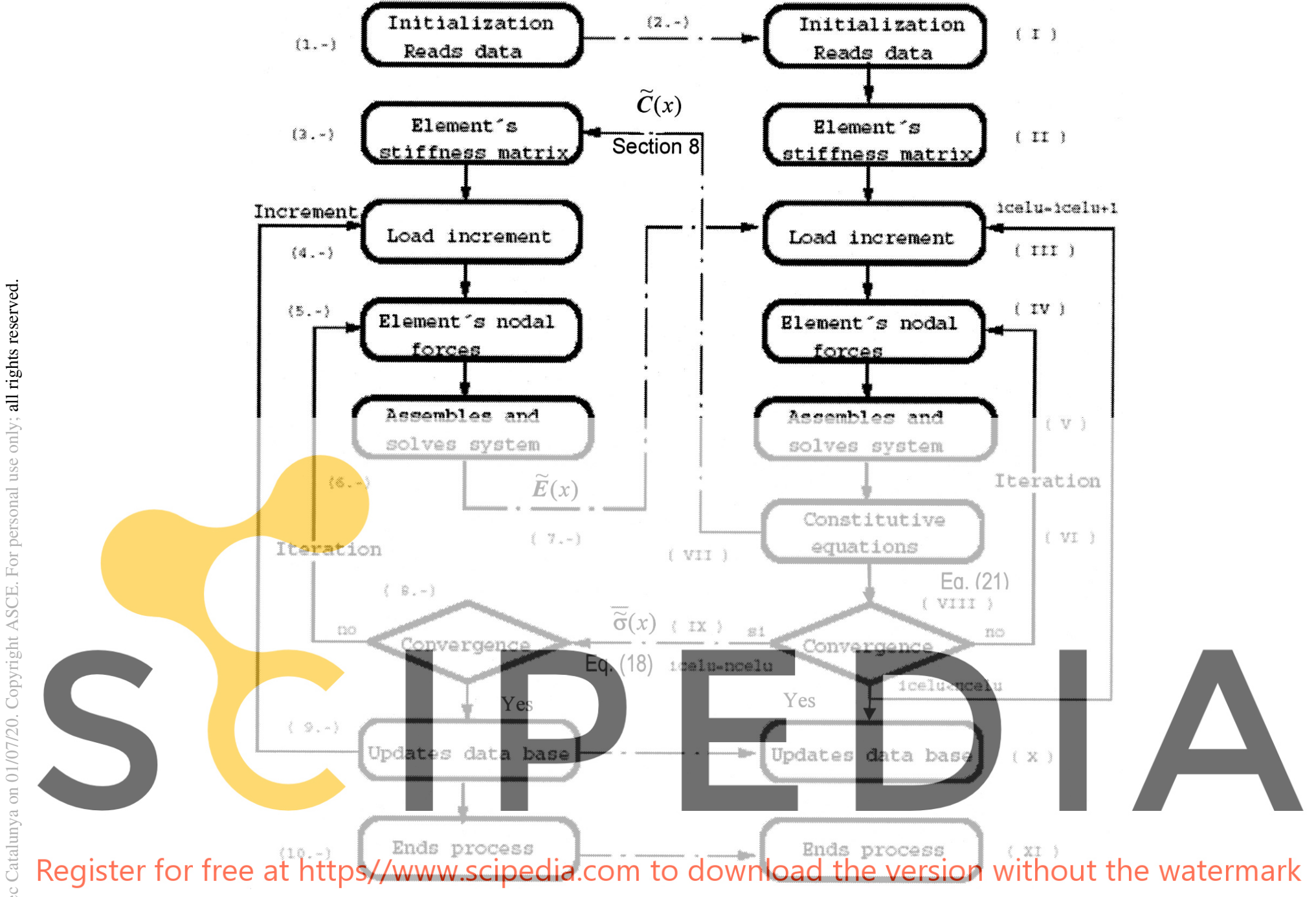

Fig. 3. Algorithm for solving composite material problems using a two-scale finite element method

\section{Macro- and Microstructural Coupling}

To solve the problems concerning composite materials by the homogenized method presented herein, a macro- and microstructural coupling is proposed that uses the finite element method on two scales (see Ghosh et al. 1996; Fish et al. 1997 for other methods that use two-scale coupling). Thus, an overall equilibrium of forces over the entire solid is imposed on the macroscopic scale, while on the microscopic scale the behavior of the composite material is solved. Thus, each point of the numerical integration of the macrostructure's finite element represents a boundaryvalue problem on the microscopic scale. This means that the governing law for the composite material behavior is a numerical constitutive equation; in this case, this macroscopic law is strain driven (because of the boundary condition imposed on the cell). The internal variables for the entire composite material correspond to all the internal variables in the whole cell domain. We must therefore simultaneously solve the overall macrostructure with many cells of composite materials, as many cells as there are numerical Gauss points in the macrostructure. The solution is obtained once all the boundary-value problems fulfill the equilibrium equations. Fig. 3 and the Appendix show the algorithms that solve the composite material problems on two scales by means of the finite element method. Their coupling implementation is carried out using a parallel virtual machine (Geist et al. 1994). This software creates a virtual data processing system constituted by one or more computers, in which several processes are handled at the same time. Therefore, one process solves the macroscopic problem, while one or more parallel processes solve the problems at the microscopic level. These processes are synchronized and information is passed from one machine to the other when necessary. Nevertheless, as each of the boundary-value problems on a microscopic scale is an independent problem, this implementation allows us to solve the cell problems in one or several processors, depending on their availability.

\section{Influence of Local Effects}

In this section, we present the influence and treatment of local effects, such as point loads and particular boundary conditions, in homogenization theory for the periodic distribution in the solids. This theory has a rigorous formulation based on the fulfillment of the following ideal suppositions: 


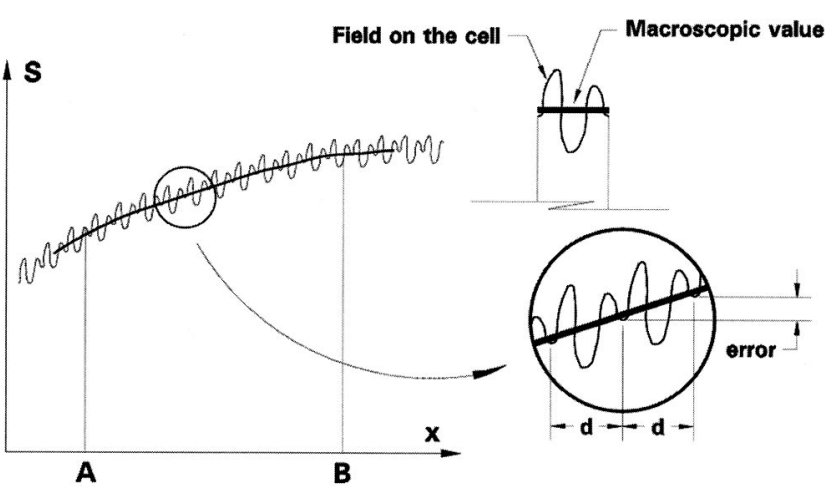

Fig. 4. Simplified representation of a quasi-periodic field function

1. The composite material has the periodic distribution of its components, which allows the virtual division in equally sized domains called $Y$ cells.

2. The cell contains the internal structure of the composite material and is very small compared to the overall structure $(Y \ll \Omega)$.

These two assumptions are contained in the local periodicity hypothesis, which demands that the stress and strains fields in the cell domain are the same as for the neighboring cells. This concept allows us to divide the problem into two scales, in which the microscopic variables fluctuate significantly, while the corre-

\section{sponding macroscopic variables change very smoothly. Conse-} quenty, the local periodicity hypothesir most of the methods based on hom of this fact can be seen in the aver: thegry, and obviously also in the
work. There are works that do no There are works that do no is the ina nain foundation of
theory. Examples
in the asymptotic
propesed in this
local periodicity variables or in the presence of local effects, such as boundary

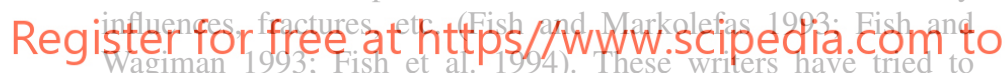
obtain the elastic problem solution at the macrostructure level by means of homogenization theory. Their formulations accept the field periodicity hypothesis on the microscopic scale, and when there are steep gradients for local effects, they introduce a perturbation term in the displacement fields. In other works, the overlapping of the high-density meshes of finite elements (multigrid technique) is used in the domain of high-gradient variables, and in these places the periodic formulation introduces these perturbation terms. These techniques are usually combined with error minimization algorithms in the finite element solution. This for-

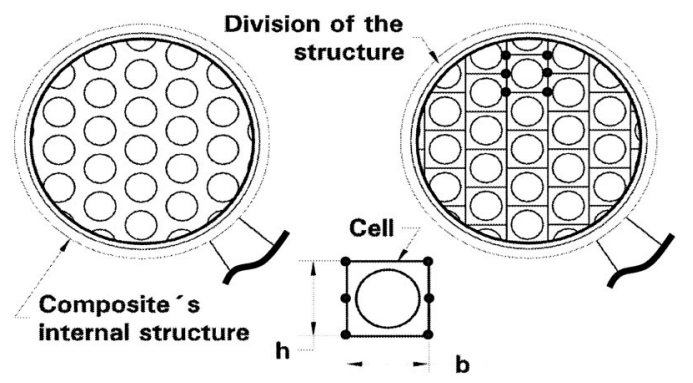

Fig. 5. Internal structure of a composite material with a square array of fibers and its cellular division into quadrilateral cells

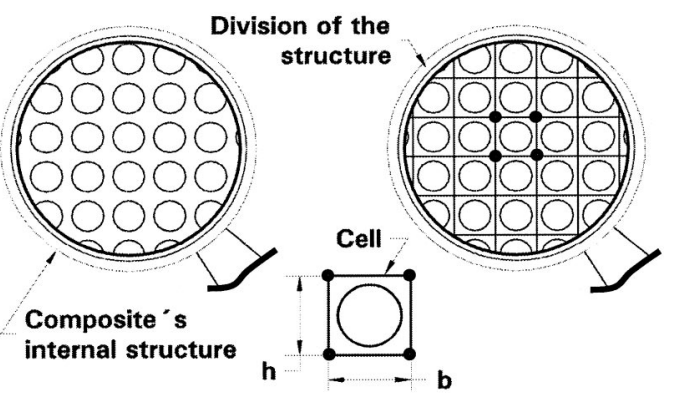

Fig. 6. Internal structure of a composite material with a hexagonal array of fibers and its cellular division into hexagonal cells

mulation increases the complexity of the problem, and it is not clear that they give better results.

A significant gradient of the macroscopic variable fields at a certain structural point involves a perturbation of these fields in the neighboring cells, which apparently contradicts the basic periodicity hypothesis. However, it should be understood that this hypothesis is only an idealization of the field's variation, which supposes that the macroscopic variable suffers slight changes. For example, let us suppose that there is a significant stress (or strain) gradient between two macrostructures, Points A and B [see Fig. 4, $\widetilde{\boldsymbol{\sigma}}_{\mathrm{A}}(x)$ and $\left.\widetilde{\boldsymbol{\sigma}}_{\mathrm{B}}(x)\right]$.

Furthermore, the homogenization theory idealizes the prob-

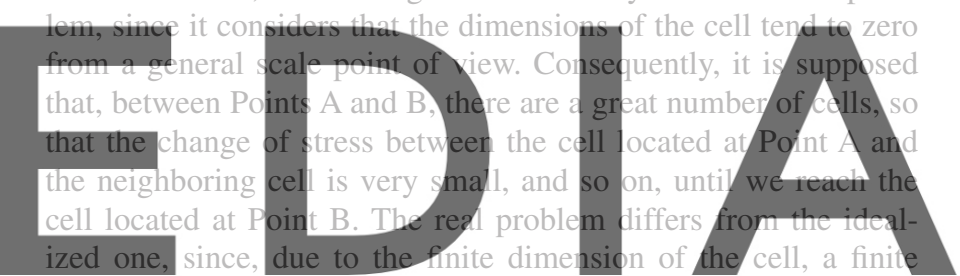
number of them may exist between $\mathrm{A}$ and B, as shown in Fig. 4. For example, on the right-hand side of the same figure, the variadawnlaad the dersianiswithadutthe awatermark derstood as the field variable error made over a period $d$ that in turn represents cell length. Let us suppose that the dimension of the cell diminishes and that the amplitude of the field variable stays constant; it is then easy to see that the error diminishes in

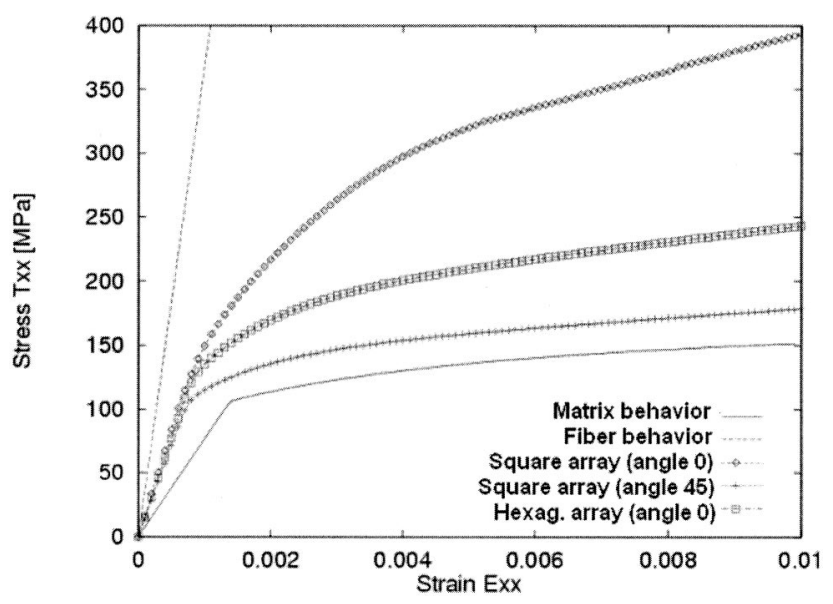

Fig. 7. Stress-strain curves for the plain strain of fiber, matrix, and square-arrayed cells, $45^{\circ}$-angle square cells, and hexagonal arrayed cells 
Table 1. Dimensions of the Cells

\begin{tabular}{|c|c|c|c|c|c|}
\hline Composite material & $\begin{array}{c}\text { Cell type } \\
\text { (number of sides) }\end{array}$ & $\begin{array}{c}\text { Fibers } \\
(\%)\end{array}$ & $\begin{array}{l}\text { Diameter of fiber } \\
\qquad(\mu \mathrm{m})\end{array}$ & $\begin{array}{c}b \\
(\mu \mathrm{m})\end{array}$ & $\begin{array}{c}h \\
(\mu \mathrm{m})\end{array}$ \\
\hline Square array & Quadrilateral & 55 & 10.0 & 11.9499 & 11.9499 \\
\hline Hexagon array & Hexagonal & 55 & 10.0 & 11.1206 & 12.8410 \\
\hline
\end{tabular}

proportion to the decrease in dimension $d$ and that, at the limit, it tends to a periodic field (see upper part of Fig. 4).

The use of the periodicity hypothesis in those places that are near to the macrostructure's boundaries has also been questioned. This topic has been studied by Sánchez-Palencia (1987), based on asymptotic developments. To analyze the boundary effect in the field variable, the writer introduces an additional displacement term, $\mathbf{u}^{1 c}$, of the microscopic order, so that the displacement field continues to be written as $\mathbf{u}^{\varepsilon}=\mathbf{u}^{0}+\varepsilon \mathbf{u}^{1}+\varepsilon \mathbf{u}^{1 c}$. Subsequent to the analysis, we can see that the influence of this additional term on the macroscopic scale is not noticeable, since the gradient of this additional term $\varepsilon \mathbf{u}^{1 c}$ vanishes very quickly. Numerical experiments show that the effect of this additional term is significant only in those cells that are on the border of the solid, when they are dumped in one period (Dumontet 1986). Nevertheless, it should be clarified that, even in this case, it is an approximation, although it is quite accurate for the remaining cells that are inside the macroscopic domain.

Consequently, if the border cell has very small dimensions in

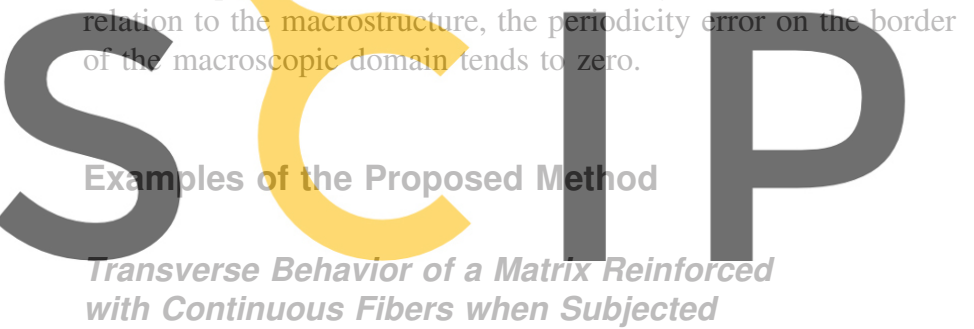

Registerffoplpręestat https//www.scipedia.com to

To validate the formulation described in the present paper, we solved the following example, presented by Jansson (1992), in which asymptotic expansion theory was used. The solid is made up of unidirectional fiber-reinforced aluminum $\left(\mathrm{Al}_{2} \mathrm{O}_{3}\right)$ matrix composite, in which the direction of the fibers is perpendicular to

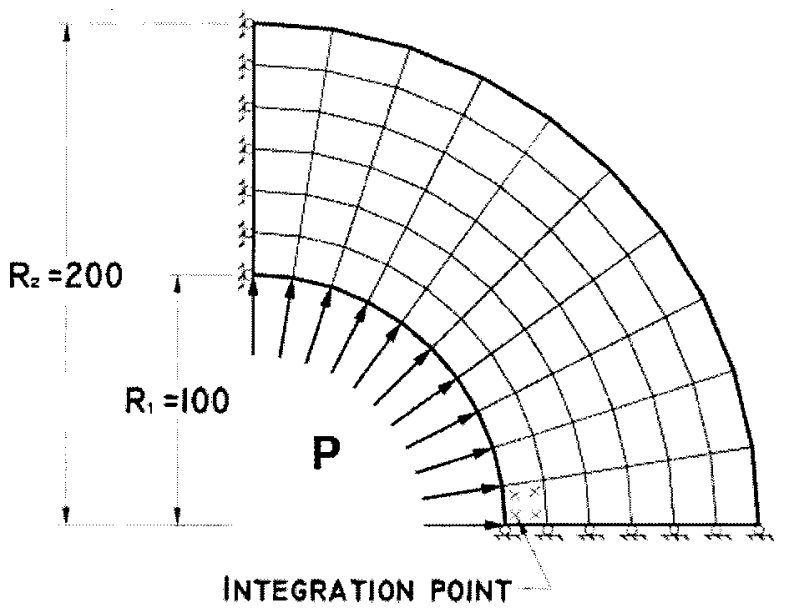

Fig. 8. Idealization of a cylindrical tube with internal pressure (macroscopic structure) the plane. In this case, we attempted to determine the composite material's behavior when subjected to uniaxial stress in a transverse direction. The problem was, in this way, reduced from three to two dimensions.

Two different cell arrays were studied. The first was a square array, as shown in Fig. 5. On the right-hand side of the same figure, the division of this array into quadrilateral cells (four periodic sides) is shown. The second array was a hexagonal array. This array and its division into hexagonal cells (six periodic sides) are shown in Fig. 6.

The composite material consists of a ductile aluminum alloy matrix reinforced with long stiff alumina fibers. The bond between fiber and matrix is very strong (perfect adherence was assumed). The fiber and matrix volume proportions are 55 and $45 \%$ respectively. The fiber's diameter was $10.0 \mu \mathrm{m}$. Consequently, the cell dimensions are shown in Table 1 .

The behavior of the fiber is assumed to be elastic and isotropic, while the aluminum matrix behavior is represented by an isotropic constitutive elastoplastic equation, which is in agree-

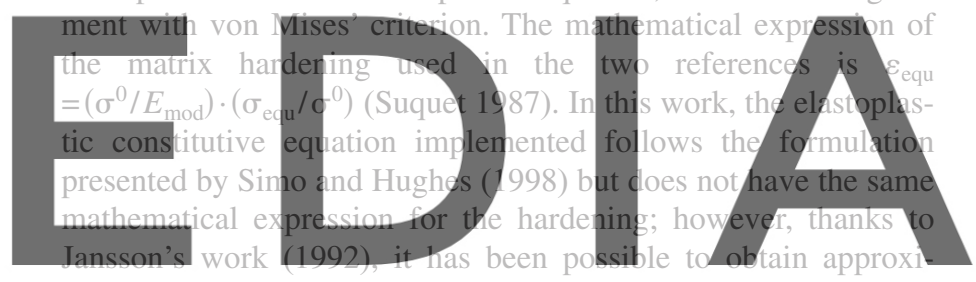

mately the same behavior for this aluminum material over a nondownload the version without the watermark nonlinear isotropic hardening were considered as $K(\alpha)=\sigma+H \alpha$ $+\left(\sigma^{\inf }-\sigma^{0}\right)(1-\exp (-\delta \alpha))$, in which the kinematic hardening $H$ $=1,000 \mathrm{MPa}$, and the difference between the initial yield stress $\left(\sigma^{0}\right)$ and the saturation stress $\left(\sigma^{\mathrm{inf}}\right)$ was $30 \mathrm{MPa}$ at a saturation velocity of $\delta=300$. The elastic properties of the component materials are presented in Table 2.

An important detail in this example is the numerical locking by the quasi-incompressible state generated in the matrix material. Jansson used nine-node isoparametric elements with selective reduced integration to avoid locking. In this work, locking was prevented by the implementation of the "B-bar" method, which is based on the mixed formulation of the finite element method in three fields: displacement, stress and strain, and fournode isoparametric elements are used.

A simple tension test may be reproduced by applying a macroscopic strain $\widetilde{E}_{x x}$ to the cell, while in a perpendicular direction the cell's strain is freed in such a way that the macroscopic stress

Table 2. Elastic Properties of the Component Materials

\begin{tabular}{|c|c|c|c|}
\hline $\begin{array}{l}\text { Composite } \\
\text { material }\end{array}$ & $\begin{array}{c}\text { Young's modulus } \\
E_{\bmod } \\
(\mathrm{MPa})\end{array}$ & $\begin{array}{c}\text { Poisson ratio } \\
(v)\end{array}$ & $\begin{array}{c}\text { Yield stress } \\
\sigma^{0} \\
(\mathrm{MPa}) \\
\end{array}$ \\
\hline Matrix $\left(\mathrm{Al}_{2} \mathrm{O}_{3}\right)$ & $68,900.0$ & 55 & 94.0 \\
\hline Fibers & $344,500.0$ & 55 & - \\
\hline
\end{tabular}


Table 3. Dimensions of the Cells

\begin{tabular}{lcccc}
\hline Composite material & $\begin{array}{c}\text { Cell type } \\
\text { (number of sides) }\end{array}$ & $\begin{array}{c}\text { Fibers } \\
(\%)\end{array}$ & $\begin{array}{c}\text { Diameter of fiber } \\
(\mu \mathrm{m})\end{array}$ & $\begin{array}{c}b \\
(\mu \mathrm{m})\end{array}$ \\
\hline Square array & Quadrilateral & 40 & 10.0 & 14.0125 \\
Hexagon array & Hexagonal & 40 & 10.0 & 14.0125 \\
\hline
\end{tabular}

$\widetilde{\sigma}_{y y}$ in this direction must be zero. Thus, the transverse behavior of the different materials is obtained. Moreover, as the squarearrayed material presents a high degree of anisotropy, the behavior of this material, when the square array is turned $45^{\circ}$, has also been found (the behavior of this material is obtained by Ghosh et al. 1996). The stress-strain curves of the matrix material, fiber material, square array cells, $45^{\circ}$-angled square array cells, and the hexagonal array cells are shown in Fig. 7. The results obtained from the behavior of these materials coincide quite closely with the behavior obtained in the aforementioned references.

\section{Thick Cylinder with Internal Pressure}

The example consists of a cylindrical tube made of a composite material (see Table 3) and subjected to an internal pressure that increases from 0 to a maximum of $100 \mathrm{MPa}$ (Ghosh et al. 1996). The symmetry of the cylinder allows us to carry out the study using a quarter of the tube's cross section. This part of the structure is divided into 60 linear quadrilateral elements, as shown in
Fig. 8, together with its dimensions and boundary conditions. This problem must be solved in the present work on both scales, and Ghosh solves the same problem using nonconventional finite elements, called Voronoi elements, to represent the microstructure, and the asymptotic expansion theory to represent the two scales.

Two cylinders were studied, each made of a different composite material: A square-array material for the first, as shown in Fig. 5, and a hexagonal array material for the second, as shown in Fig. 6. In both cases, the composite material was $40 \%$ fiber and $60 \%$ matrix. The mechanical properties of these component materials are identical to those in the previous example (Table 2). The dimensions of the cells are shown in Table 3.

In this case, in order to solve the problem for both scales, it is necessary to solve the macrostructure and 240 cells (60 elements with 4 integration points each) for each load increment.

The result obtained with the first composite material can be seen in Fig. 9, which shows four graphs of the macrostructure,

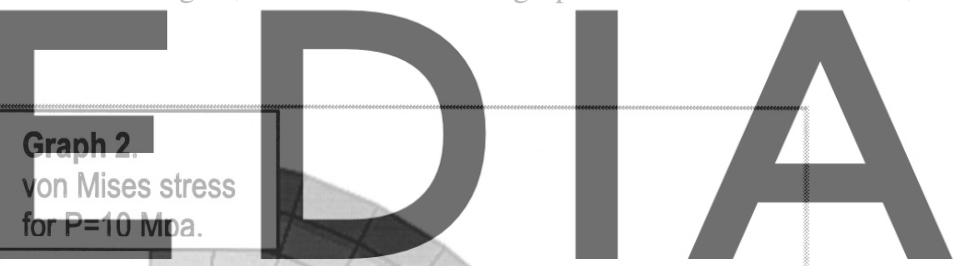

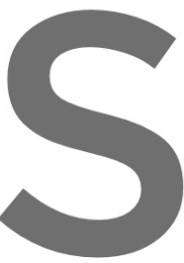

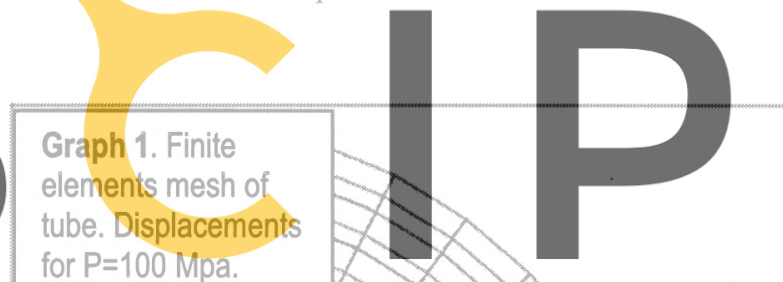

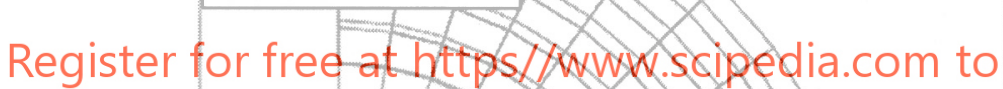
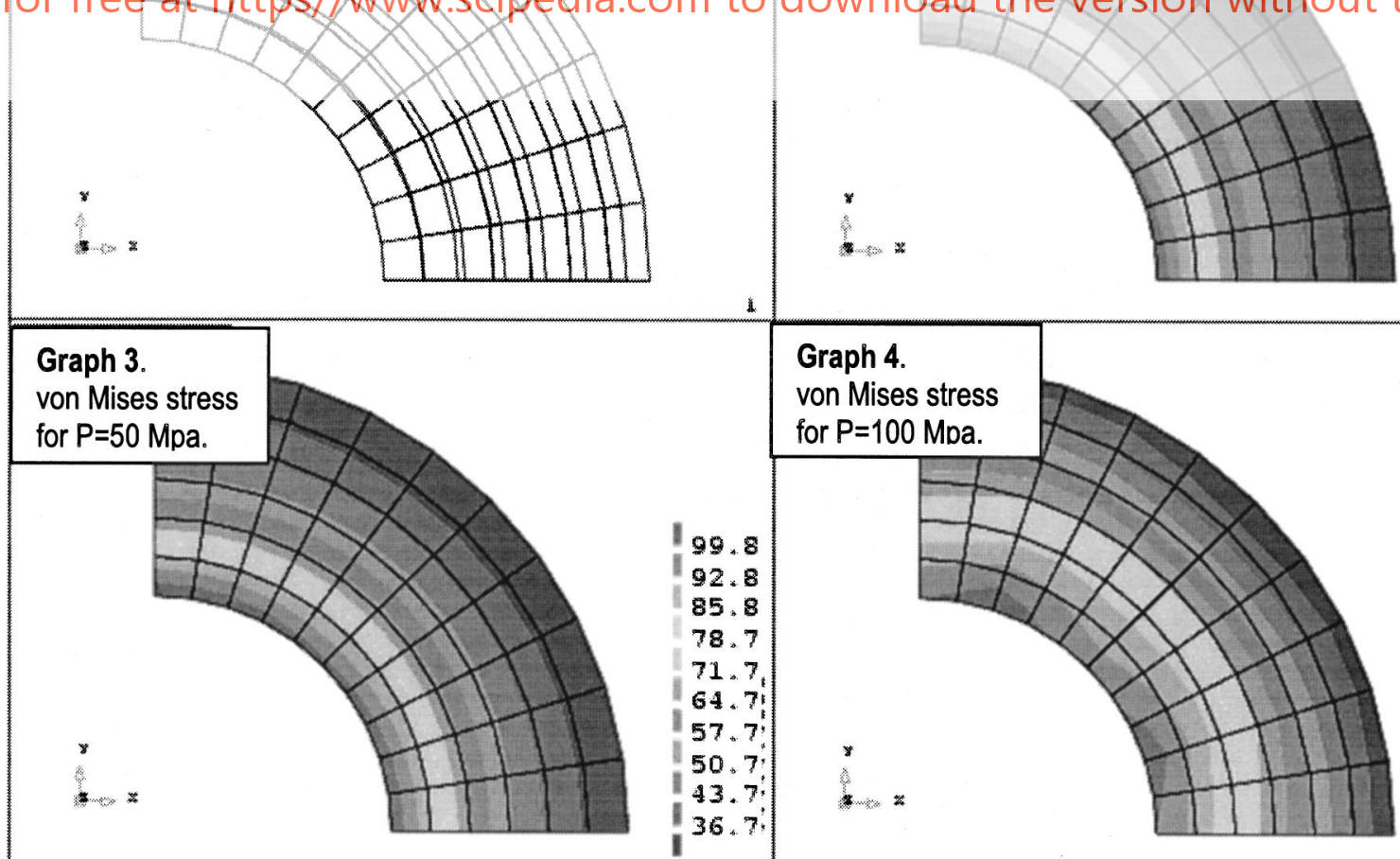

15

147

139

132

124

117

109

102

194.7

87.2

Fig. 9. von Mises stress graphs for the composite macrostructure at three different pressure levels 


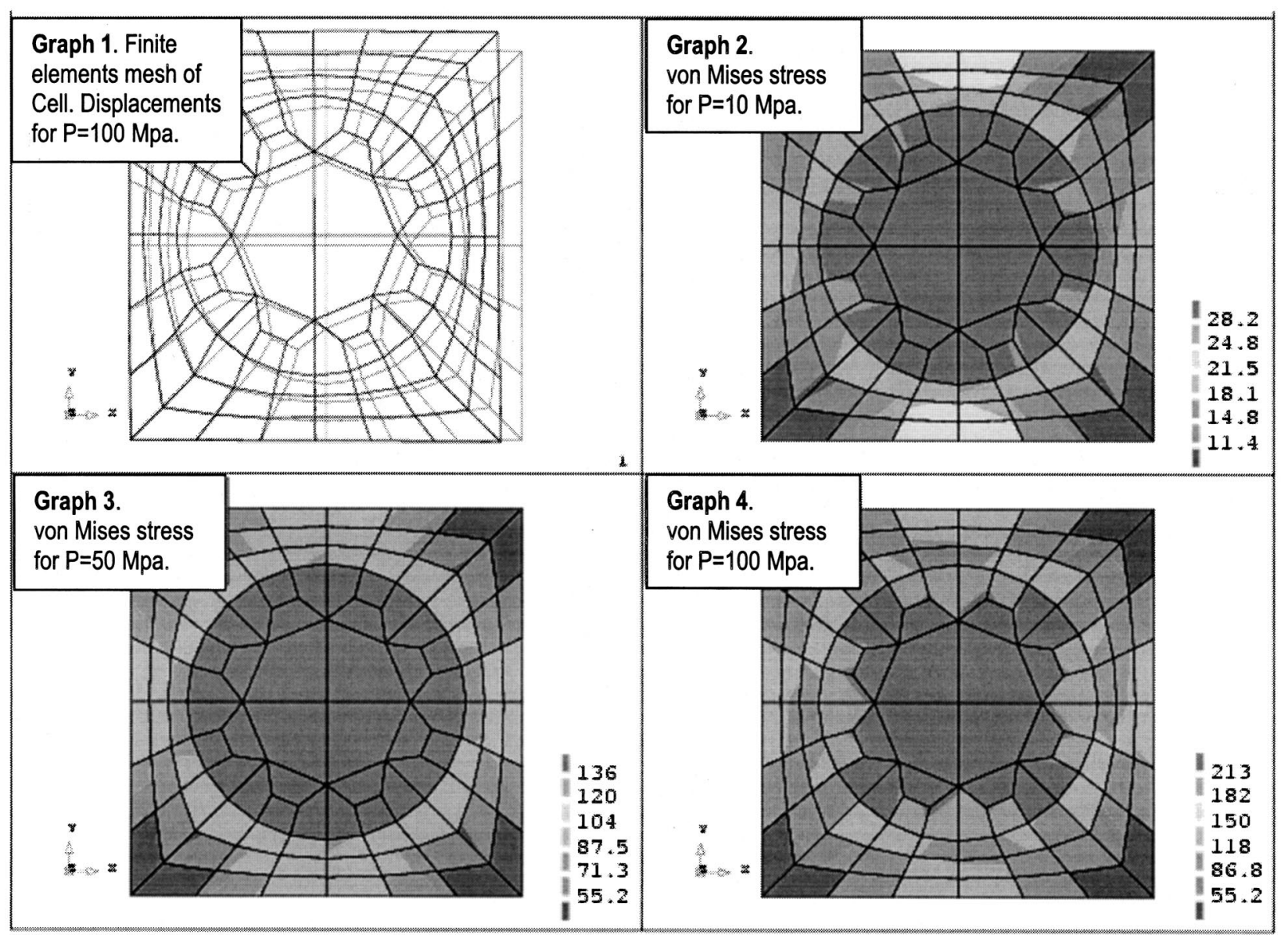

Fig. 10. von Mises stress graphs for the microstructure at three different pressure levels at the first integration point of the first finite element (see Fig. 8)

and Fig. 10, which shows four graphs of the microstructure that correspond to the first integration point of the first finite element (indicated in Fig. 8). In each of these two groups of figures (macrostructure and microstructure), Graph 1 represents the division of the structure into finite elements and mesh displacements at a pressure of $100 \mathrm{MPa}$. In Graph 2, the field of stress is presented according to the von Mises plastic yield criterion when the pressure applied is $10 \mathrm{MPa}$. In this case, the whole domain of the tube is in the linear elastic range. The distribution of the stress on the tube corresponds to the isotropic material in an approximate manner, since the field of the stress is practically uniform in the circumferential direction. Graph 3 presents the von Mises stress when the pressure applied reaches $50 \mathrm{MPa}$; at this pressure level, the nonlinear process begins in a few integration points. Finally, in Graph 4, the von Mises stress is shown when the pressure reaches $100 \mathrm{MPa}$. In this case, part of the composite material is in the nonlinear range and anisotropy is observed, which produces

Table 4. Properties of the Materials

\begin{tabular}{lcccc}
\hline & $\begin{array}{c}\text { Young } \\
\text { modulus } \\
E_{0}\end{array}$ & $\begin{array}{c}\text { Poisson } \\
\text { coefficient } \\
(v)\end{array}$ & $\begin{array}{c}\text { Tension } \\
\text { strength } \\
f_{t} \\
\left(\mathrm{~N} / \mathrm{mm}^{2}\right)\end{array}$ & $\begin{array}{c}\text { Compression } \\
\text { strength } \\
\left.\mathrm{mm}_{c}\right)\end{array}$ \\
\hline Brick & 20,000 & 0.15 & 5.0 & 15.0 \\
Cement mortar & 2,000 & 0.20 & 1.5 & 15.0 \\
\hline
\end{tabular}

stress concentration in parts of the domain. These results coincide quite closely with those reported (Ghosh et al. 1996) for a pressure of $100 \mathrm{MPa}$ (Figs. 9 and 10), in spite of significant differences in the kind of formulation, because they use Voronoi's finite elements. The results obtained using the hexagonal cells are similar to those already obtained using the square cell shown in Figs. 9 and 10. This implies that a composite material with a hexagonal array is also anisotropic in a nonlinear range.

\section{Homogenized Masonry as a Composite Material}

An example is presented in which the masonry of traditional brick is studied as a composite material. Molins (1996) carried out a review of some of the most relevant ways of characterizing these kinds of materials, which allows the difficulties and limitations of existing models to be verified. The example presented consists of a masonry wall under a shear load laboratory test, in which a micromodel is formulated to obtain the structural masonry's behavior (Lourenço 1996; Zijl et al. 1997). The properties of the brick and the mortar are shown in Table 4. Perfect adherence between the mortar and brick is assumed.

Fig. 11 shows the fracture distribution of two walls tested under shear loads. Each wall measures $990 \mathrm{~mm}$ along the base by $1,000 \mathrm{~mm}$ in height and has a window in its interior. The dimensions of the bricks used in the wall are $210 \times 52 \times 100 \mathrm{~mm}$. The bricks are bonded together using $10 \mathrm{~mm}$ of cement mortar. The laboratory test consists in fixing the base of the wall and applying 

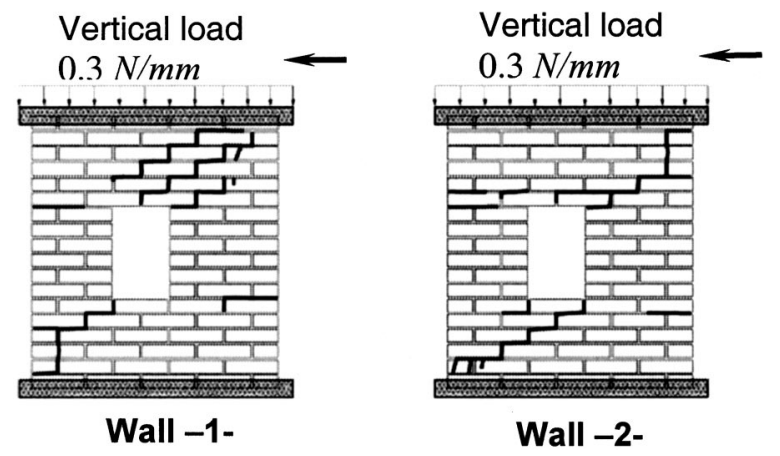

Fig. 11. Two wall masonry fracture distribution under shear load

a distributed vertical load of $0.3 \mathrm{~N} / \mathrm{mm}$ to its upper part. Once the load is applied, a horizontal load is imposed on the top level under displacement control.

The numerical simulation of the behavior of each component material was carried out using isotropic damage models (Oliver et al. 1990). Two different isotropic damage constitutive models were used: In the first, the component materials were subjected to exponential softening, and in the second, the fracture energy of the mortar was increased, representing the friction between mortar and brick after the mortar fracture. This introduced an artificial strength threshold for friction. The cell that characterized the composite consisted of a simple brick, and a mortar layer that was $5 \mathrm{~mm}$ thick surrounding it.

The wall was divided into 66 quadrilateral elements. Four Gaussian numerical integration points were used in each finite element. Consequently, the solution of the macrostructure for each of the load increments was obtained in parallel with the 264 cells used.

The homogenization theory gives information on the results in each of the scales. Therefore, we preferred to illustrate the degradation or damage that took place in the wall (see Fig. 12) by representing the average of the damage variable in each of the cells. Graph 1, in Fig. 12, presents the discretization of the wall into 66 quadrilateral finite elements. In Graphs 2, 3, and 4 in the same figure, the average of the damage variable is presented. The damage variable was drawn on the deformed wall, as a consequence of the displacement level applied in each case. Graph 2 represents the wall damage when the horizontal displacement reached $1.50 \mathrm{~mm}$ in the upper part of the wall. As shown, the damage of the wall began in two opposite window corners, produced by the shear stresses, while tension stresses resulted in damage in the lower right-hand area and in the upper left-hand area of the wall. Graph 3 in Fig. 12 represents the damage when the horizontal displacement reached $2.75 \mathrm{~mm}$ in the upper part of the wall. In Graph 4, the displacement imposed was $4.0 \mathrm{~mm}$. One can observe the crushing damage in the lower left-hand area

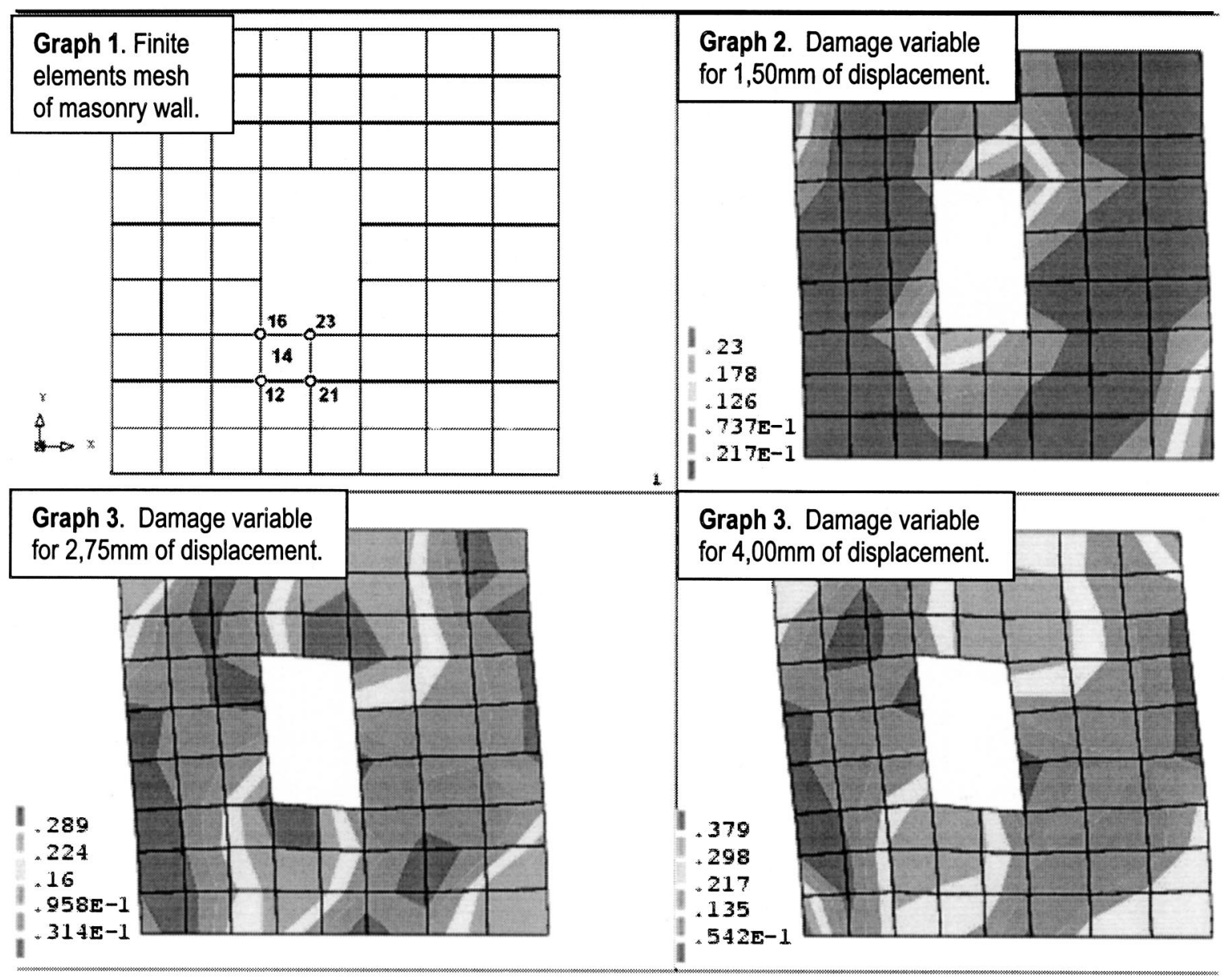

Fig. 12. Wall's deformed shape and damage level for three different load increments 
Graph 1. Finite

elements mesh of Cell.

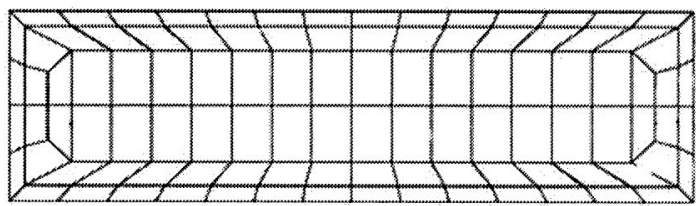

*

$* *$
Graph 2. Cell deformed mesh for

$1,5 \mathrm{~mm}$ of displacement in the upper part of wall (see Fig. 12).

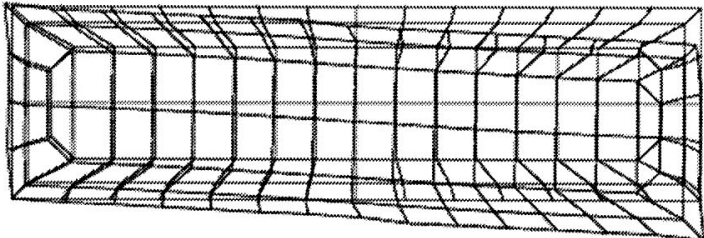

4. $:$

i

Graph 3. Shear strain for
$1,5 \mathrm{~mm}$ of displacement in the
upper part of wall (see Fig. 12).

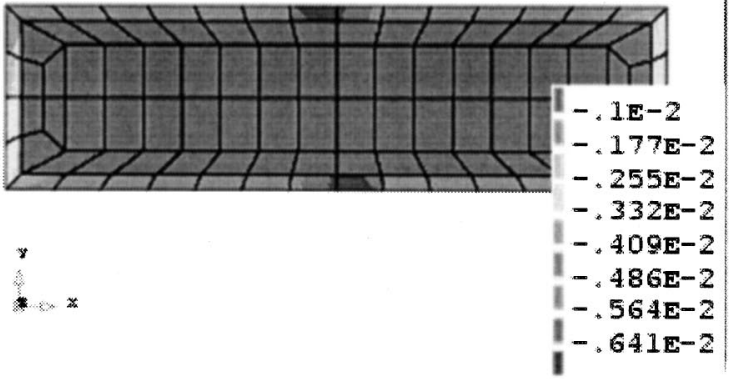

Graph 4. Shear stress for $1,5 \mathrm{~mm}$ of displacement in the upper part of wall (see Fig. 12).

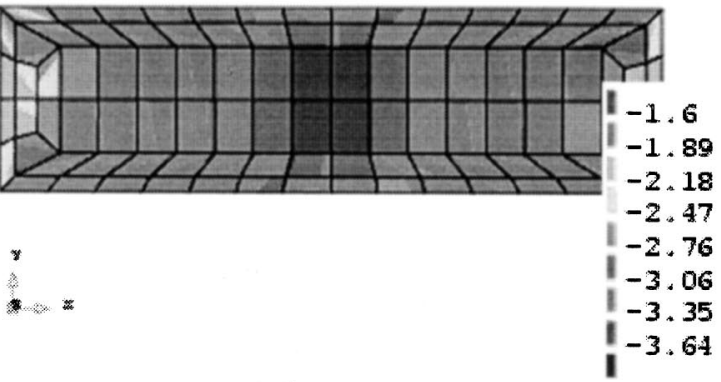

Fig. 13. Wall cell representation of the third integration point of the fourteenth finite element (see Fig. 12), for a displacement of $1.5 \mathrm{~mm}$ in the upper part of the wall: (1) finite element discretization, (2) deformed mesh, (3) shear strain, and (4) shear stress

and in the upper right-hand area of the wall. In this example, the tension cracks took place in the mortar because it was the weakest component, while the crushing by compression took place in the two compounds of the composite material (brick and mortar).

As a result of homogenization theory, the two-scale solution problem also provides a wealth of information on mechanical behavior at the microscale level. As explained above, the behavior of the discretized composite material is obtained starting from the macrolevel mechanical solution for each integration point, so that

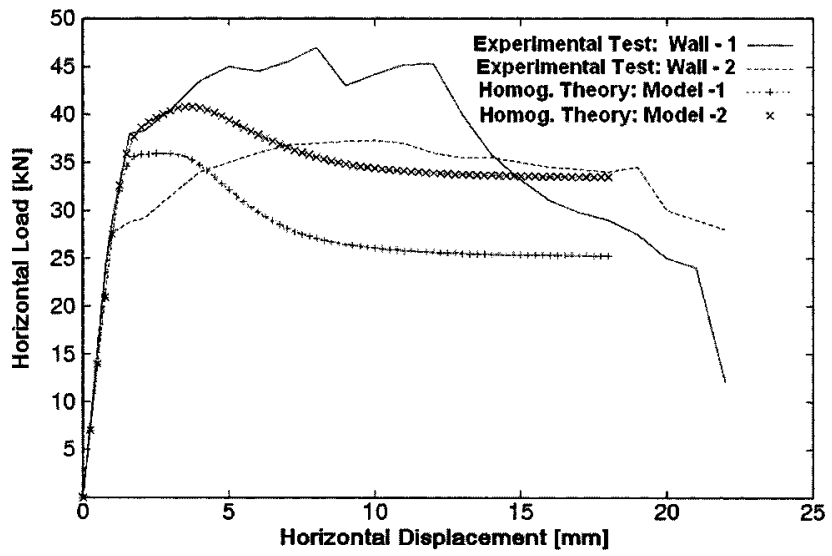

Fig. 14. Numerical and experimental comparison of horizontal loaddisplacement behavior each one of these points represents a cell in the microlevel domain. Consequently, the fields of the microscopic variables are obtained in each of these points. For example, Fig. 13 presents four graphs of the cell of the composite material, that corresponds to the third integration point of the fourteenth element of the macrostructure (see Fig. 12), when the displacement applied is $1.5 \mathrm{~mm}$. Graph 1 in Fig. 13 shows the cell domain, subdivided into 104 quadrilateral finite elements of four nodes. Graph 2 of the same figure shows the deformed mesh. Graph $\mathbf{3}$ indicates the field of shear strain in the domain of the cell, which shows the mortar strain concentration, and Graph $\mathbf{4}$ presents the cell field of shear stress.

Fig. 14 shows the curves of the horizontal load measured in the base of the wall, against the displacement imposed on the upper part of the wall. The first numerical model's solution using the homogenization theory coincides with the second model and experimental results in the elastic range, but in the nonlinear range the strength deteriorates rapidly due to mortar failure. In the second numerical model, the solution matches the real results better in the nonlinear range. In this case, the fracture energy in the mortar was increased to simulate artificial friction.

\section{Conclusion}

In this paper, the homogenization theory for the periodic internal structure of nonlinear materials is formulated in two scales (micro and macro). This formulation is based on the homogenized strain 
tensor related to the change of the periodicity vector and of the homogenized stress tensor obtained by means of the classic average theory.

The coupling between the micro- and macroscales was carried out by means of the finite element method; a parallel strategy was used to simultaneously solve the cells on the microscale and the composite material on the macroscale. Each of the basic cells at the microscale level was assigned to each numerical integration point on the macroscale.

Classic constitutive equations, such as damage, plasticity, viscoelasticity, viscoplasticity, etc., were taken into account when defining the microscale's mechanical behavior. Internal variables were stored at each point at the microscale level.

To prevent the steep gradient in the macroscopic field variables that is produced by local boundary effects, a local refinement of the finite element mesh was performed. In this way, we were able to maintain the periodic condition on the boundaries of the cells near to the perturbation.

A quasi-tangent inelastic tensor at the macroscale level was used to define the inelastic constitutive law in the whole cell at each numerical integration point on the macroscale. The elastic constitutive tensor was obtained using the perturbation technique at the same macroscale level.

The homogenization theory, in general, and the method presented here, in particular, give good results in comparison to other approaches such as the mixing theory (Car et al. 2002), although these comparisons highlight the need for improving effectiveness in the solving of large problems.

\section{Appendix. Two-Scales Algorithm for Studying the Behavior of Composite Materials Using the Finite Element Method}

The algorithm in Fig. 3 describes the procedure proposed for the analysis of the homogenized composite material using finite elements on two scales.

1. The global program (macroscopic scale) starts with the matrix initialization tasks; it reads the information on the macrostructure and the local program's address to solve the microscopic scale problem.

2. Computation of the elastic homogenized constitutive tensor $\widetilde{\mathbf{C}}(x)$ through the local program (microscopic scale).

- The local program initializes the database with the number of cells and reads the microstructure's information.

- The local program calculates the stiffness matrix for the cell using the homogenized constitutive tensor.

- The load increment is applied. In this case, the solution is reached by imposing a preset macroscopic strain $\tilde{\mathbf{E}}(x)$ [see Eq. (29)]. For this purpose, it fulfills the tasks that follow.

- The nodal forces are obtained.

- The microstructure's system of equations is assembled, and then solved under periodic boundary conditions.

- The stresses on the microstructure are obtained using the constitutive equation [Eq. (21)]. In this case, the composite material displays elastic behavior. Subsequently, the homogenized stress tensors $\tilde{\boldsymbol{\sigma}}(x)$ are obtained [Eq. (18)].

- The elastic homogenized constitutive tensor is calculated by means of the perturbation procedure $\widetilde{\mathbf{C}}(x)$ (see section entitled, "Determination of the Constitutive Tensor for
Composite Materials"). The result is sent to the global program.

3. The stiffness matrix for each finite element in the global structure is obtained (macroscopic scale).

4. The new load increment is applied.

5. The nodal forces for each finite element are computed.

6. The global structure's system of equations is computed and solved.

7. The acceptable stress level at each point in the macrostructure is verified by means of the constitutive equation. This task is carried out by returning to the local program, and the information on the homogenized strains $\tilde{\mathbf{E}}(x)$ at each integration point of the macroscopic scale is sent from the global program to the local program:

- The local program solves each of the cells sequentially. This task reads the information on the microscopic problem (cell) from the database and applies it as a load strain increment $\dot{\widetilde{\mathbf{E}}}(x)$.

- The nodal forces are obtained.

- The system equation at the microstructure level is assembled and solved under periodic boundary conditions.

- The acceptable microscopic stress levels $\boldsymbol{\sigma}(y)$ are verified by means of the respective constitutive equations for each single material. If they cannot be verified, they should be corrected. After this step, the cell's homogenized stress $\widetilde{\boldsymbol{\sigma}}(x)$ and constitutive tensor $\widetilde{\mathbf{C}}(x)$ are obtained.

- The balance condition is verified in the cell domain. If it is not fulfilled, the balance condition must return to the prior step and the convergence is reached following an iterative strategy (Newton-Raphson procedure). The stress is then obtained for the macroscopic scale $\widetilde{\boldsymbol{\sigma}}(x)$,

- When all the cells have been solved, the information on the homogenized stress and constitutive tensor are transmitted to the global program.

8 The global program receives the stresses $\widetilde{\boldsymbol{\sigma}}(x)$ and the constitutive tensors $\widetilde{\mathbf{C}}(x)$ in each integration point from the local program. The subsequent step verifies the balance of the forces in the global structure domain (macroscopic scale). If the balance condition is not reached, it returns to Step 5 and convergence is reached by means of successive iterations (quasi-Newton's method).

9 The databases of the global program are updated when the problem converges in the macroscopic scale. If there are further increments, it returns to Step 4.

- The databases of the local program are updated simultaneously (for all cells).

10 The global program ends.

- The local program ends.

\section{References}

Anthoine, A. (1995). "Derivation of the in-plane characteristics of masonry through homogenization theory." Int. J. Solids Struct., 32(2), $137-163$.

Aravas. (1995).

Bensoussan, A., Lions, J. L., and Papanicolaou, G. (1978). Asymptotic analysis for periodic structures, North-Holland, Amsterdam.

Car, E., Zalamea, F., Oller, S., Miquel, J., and Oñate, E. (2002). "Numerical simulation of fiber reinforced composite materials: Two procedures.” Int. J. Solids Struct., 39(7), 1967-1986. 
Crisfield, M. (1980). Numerical methods for nonlinear problem, Pineridge, Swansea, U.K.

Dennis, J. E., and More, J. J. (1977). "Quasi-Newton methods, motivation and theory." SIAM Rev. 19(1), 46-89.

Devries. (1989).

Dumontet, H. (1986). "Boundary layer stresses in elastic composites." Local effects in the analysis of structures, Elsevier, Amsterdam.

Duvaut, G. (1976). "Analyse fonctionnelle et mécanique des milieux continus." Theoretical and applied mechanics, W. Roiter, ed., NorthHolland, Amsterdam, The Netherlands, 119-132.

Dvorak, G. J., Bahei-El-Din, Y. A., and Wafa, A. M. (1994). "Implementation of the transformation field analysis for inelastic composite materials." Comput. Mech., 14, 201-228.

Fish, J., and Markolefas, S. (1993). "Adaptive s-method for linear elastostatics." Comput. Methods Appl. Mech. Eng., 104, 363-396.

Fish, and Shek. (1999).

Fish, J., and Wagiman, A. (1993). "Multiscale finite element method for a locally nonperiodic heterogeneous medium." Comput. Mech., 12, 164-180.

Fish, J., Nayak, P., and Holmes, M. H. (1994). "Microscale reduction error indicators and estimators for a periodic heterogeneous medium." Comput. Mech., 14, 323-338.

Fish, J., Shek, K., Pandheeradi, M., and Shephard, M. S. (1997). "Computational plasticity for composite structures based on mathematical homogenization: Theory and practice." Comput. Methods Appl. Mech. Eng., 148, 53-73.

Geist, A., et al. (1994). Parallel virtual machine: A user's guide and tutorial for networked parallel computing, Massachusetts Institute of Technology, Cambridge, Mass.

Ghosh, S., Lee, K., and Moorthy, S. (1996). "Two-scale analysis of heterogeneous elastic-plastic materials with asymptotic homogenization and Voronoi cell finite element model." Comput. Methods Appl. Mech. Eng., 132, 63-116.

Jansson, S. (1992). "Homogenized nonlinear constitutive properties and local stress concentrations for composites with periodic internal structure." Int. J. Solids Struct., 29 (17), 2181-2200.

Lee, K., Moorthy, S., and Ghosh, S. (1999). "Multiple scale computational model for damage in composite materials." Comput. Methods Appl. Mech. Eng., 172, 175-201.

Lene, F. (1986). "Damage constitutive relations for composite materials." Eng. Fract. Mech., 25, 713-728.

Lourenço, P. (1996). Computational strategies for masonry structures, Delft University Press.
Molins, C. (1996). Structural analysis of historical constructions, CIMNE, Barcelona.

Oliver, J., Cervera, M., Oller, S., and Lubliner, J. (1990). "Isotropic damage models and smeared crack analysis of concrete." Proc., 2nd Int. Conf. on Analysis and Design of Concrete Structures, Vol. 2, 945-958.

Sanchez-Palencia, E. (1980). Vol. 127, "Nonhomogeneous media and vibration theory." Lecture notes in physics, Springer, Berlin.

Sanchez-Palencia, E. (1987). "Boundary layers and edge effects in composites." Homogenization techniques for composite media, Springer, Berlin, 121-192.

Simo, J. C. and Hughes, T. J. R. (1998). Computational inelasticity, Springer, New York.

Suquet, P. M. (1982). "Plasticité et homogénéisation." PhD thesis, Univ. Pierre et Marie Curie, Paris 6.

Suquet, P. M. (1987). "Elements of homogenizations for inelastic solid mechanics." Homogenization techniques for composite media, Springer, Berlin, 193-279.

Zalamea. (2002).

Zalamea, F. (2001). "Composite modeling by means of the homogenization theory." $\mathrm{PhD}$ thesis, Technical Univ. of Catalonia, Spain (in Spanish).

Zalamea, F., Miquel Canet, J. and Oller, S. (1998). "Treatment of composite materials based on the homogenization method." Proc., 4th World Congress on Computational Mechanics, E. Oñate, S. Idelsohn, and E. Dvorkin, eds., CD-ROM, CIMNE, Barcelona.

Zalamea, F., Miquel Canet, J., and Oller, S. (1999a). "Teoría de homogeneización para el análisis de materiales compuestos con estructura interna periódica." Proc., 4th Conf. on Numerical Methods in Engineering, R. Abascal, J. Dominguez, and G. Bugeda, eds., SEMNI CD-ROM.

Zalamea, F., Miquel, J., and Oller, S. (1999b). "Un mätodo en doble escala para la simulación de materiales compuestos." 3rd Natl. Conf. on Composite Materials, "MATCOMP 99," A. Corz and J. M. Pintado, eds., Materiales Compuestos 99-AEMAC, 381-393.

Zalamea, F., Miquel, J., and Oller, S. (2000). "A double-scale method for simulating of periodic composite materials." European Congress on Computational Methods in Applied Sciences and Engineering, ECOMAS 2000-COMPLAS VI, E. Oñate and R. Owen, eds., CDROM, CIMNE, Barcelona.

Zienkiewicz, O., and Taylor, R. (1991). The finite element method, Vols. I and II, McGraw-Hill, London.

Zijl, et al. (1997). 